\section{AN INTEGRAL VERSION OF THE BROWN-GITLER SPECTRUM}

BY

DON H. SHIMAMOTO

\begin{abstract}
In this paper, certain spectra $B_{1}(k)$ are studied whose behavior qualifies them as being integral versions of the Brown-Gitler spectra $B(k)$. The bulk of our work emphasizes the similarities between $B_{1}(k)$ and $B(k)$, shown mainly using the techniques of Brown and Gitler. In analyzing the homotopy type of $B_{1}(k)$, we provide a free resolution over the Steenrod algebra for its cohomology and study its Adams spectral sequence. We also list conditions which characterize it at the prime 2. The paper begins, however, on a somewhat different topic, namely, the construction of a configuration space model for $\Omega^{2}\left(S^{3}\langle 3\rangle\right)$ and other related spaces.
\end{abstract}

Introduction. In a paper published in 1973, E. H. Brown, Jr., and S. Gitler described a procedure by which one might conceivably find new characteristic classes for smooth $n$-dimensional manifolds [3]. Their approach was based on examining the way that certain cohomology operations act on the stable normal bundle. Much of the motivation for their work stemmed from the problem of immersing manifolds in Euclidean space. For example, suppose that $M$ is an $n$-manifold and let $U$ denote the mod 2 Thom class of its stable normal bundle. From standard properties of the Steenrod squares, it follows that if $\mathrm{Sq}^{i} U \neq 0$, then $M$ cannot be immersed in $R^{n+i-1}$.

Brown and Gitler's idea was to take all those elements of the mod 2 Steenrod algebra which universally vanish on the Thom classes of $n$-manifolds and use these "primary" operations as the foundation for a coherent system of higher order cohomology operations which might then prove useful, say, to detect nonimmersions.

As it turns out, these higher order operations also universally vanish on $n$-manifolds, and the attempt to produce new characteristic classes failed. The story has a happy ending, though, as Brown and Gitler were able to use their analysis to deduce the existence of a family of spectra $B(k)$. Since then, the Brown-Gitler spectra have played an important part in the development of the immersion problem and have also appeared, startlingly, in certain constructions involving the May filtration of the double loop space $\Omega^{2} S^{3}$.

This paper partially arswers the questions of what happens if one tries to repeat Brown and Gitler's anaiysis, but only for $Z$-orientable $n$-manifolds. Our main results

Received by the editors March 7, 1983.

1980 Mathematics Subject Classification. Primary 55P42; Secondary 55P35, 55S10, 55S45, 57R19.

Key words and phrases. Brown-Gitler spectrum, Steenrod algebra, iterated loop space, Thom spectrum, $\Lambda$-algebra, Adams spectral sequence, orientability of manifolds, Postnikov tower. 
concern a family of spectra $B_{1}(k)$ having the following properties:

(0.1) $H^{*}\left(B_{1}(k)\right)=A / A\left\{\mathrm{Sq}^{1}, \chi\left(\mathrm{Sq}^{i}\right) \mid i>k\right\}$ as $A$-modules. (Here, and from now on, homology and cohomology should be taken with coefficients in $Z_{2}$, the integers mod 2, unless otherwise noted. Also, $A$ denotes the mod 2 Steenrod algebra, and $\chi$ : $A \rightarrow A$ is the canonical antiautomorphism.)

(0.2) Let $Z_{\hat{2}}=\lim Z_{2^{\prime}}$ denote the 2-adic integers. There is a map $j: B_{1}(k) \rightarrow K\left(Z_{\hat{2}}\right)$ such that, for any CW complex $X$, the induced map of homology theories $j_{*}$ : $B_{1}(k)_{n}(X) \rightarrow H_{n}\left(X ; Z_{\hat{2}}\right)$ is surjective, provided that $n<2 k+2$.

$\left(0.2^{\prime}\right)$ All $Z$-orientable, closed $n$-manifolds are $B_{1}([n / 2])$-orientable. That is, given such a manifold $M$, let $U_{7}: T(\nu) \rightarrow K\left(Z_{\hat{2}}\right)$ represent the Thom class of its stable normal bundle. Then there exists $U_{B}: T(\nu) \rightarrow B_{1}([n / 2])$ such that $j U_{B}=U_{7}$.

We shall also prove that $(0.1)$ and $(0.2)$ characterize $B_{1}(k)$, up to homotopy 2-equivalence; further arguments show that $(0.2)$ and $\left(0.2^{\prime}\right)$ are essentially equivalent. Moreover, $B_{1}([n / 2])$ has the smallest possible mod 2 cohomology for any spectrum possessing the orientability property $\left(0.2^{\prime}\right)$, provided that $n$ is not divisible by 4 .

A definition for a family of spectra satisfying (0.1) was first proposed by Mahowald. We shall base our presentation on Mahowald's definition, but, in an appendix, we discuss another way in which $B_{1}(k)$ could be defined.

Our viewpoint is strongly influenced by a desire to display $B_{1}(k)$ as an integral version of the Brown-Gitler spectrum $B(k)$. Brown and Gitler originally constructed $B(k)$ by building a generalized Postnikov tower for it, or, really, the Pontrjagin dual of such a tower. Normally, one starts with a known spectrum and then builds a Postnikov tower for it. Brown and Gitler faced the difficulty of trying to use a Postnikov tower for $B(k)$ in order to prove that it actually exists. Their construction was quite complicated.

However, in a seemingly unrelated development, the theory of Thom spectra was attracting considerable attention, largely due to the techniques and examples of Mark Mahowald (see [15] for an overview of the subject). Proceeding on the basis of some of Mahowald's cohomological calculations, R. Cohen [9] and Brown and Peterson [6] made the discovery that the Brown-Gitler spectra could be realized as certain Thom spectra involving the May filtration of $\Omega^{2} S^{3}$. Because of the complexity of Brown and Gitler's original work, it was surprising, but comforting, to find that the spectra $B(k)$ could be obtained by these more natural geometric means. Not surprisingly, it seems quite difficult to use these geometric realizations of $B(k)$ in direct proofs of the sort of deeper properties that Brown and Gitler obtained via their Postnikov analysis.

The spectra $B_{1}(k)$ that we shall study here will be defined geometrically, following Mahowald, as Thom spectra (completed at 2). The details of Mahowald's construction have not yet appeared in print, so, in the appendix, we describe an independent (non-Thom spectrum) approach to defining $B_{1}(k)$. At any rate, there is no question that $B_{1}(k)$ exists. Even so, one might try to mimic Brown and Gitler's analysis in order to obtain deeper homotopy results. Indeed, this is the approach we will take: a recurrent theme in this paper is to start with properties about the Brown-Gitler spectrum $B(k)$ and then prove suitable analogues for $B_{1}(k)$. 
Brown and Gitler's starting point was the construction of an explicit $A$-free resolution of $H^{*} B(k)$. Correspondingly, we shall define $A$-modules $C_{q}$ and differentials $d: C_{q} \rightarrow C_{q-1}$ such that

(0.3) $\cdots C_{q} \stackrel{d}{\rightarrow} C_{q-1} \rightarrow \cdots \rightarrow C_{0} \rightarrow H^{*} B_{1}(k) \rightarrow 0$ is an $A$-free resolution of $H^{*} B_{1}(k)$.

Using this resolution and the Adams spectral sequence then yields:

$(0.4 \mathrm{a}) \pi_{0}\left(B_{1}(k)\right)=Z_{\hat{2}}$.

(0.4b) If $1 \leqslant q \leqslant 4 k+2$, then

$\pi_{q}\left(B_{1}(2 k)\right)=\pi_{q}\left(B_{1}(2 k+1)\right)=\left(\Lambda\left\{\lambda_{1}, \lambda_{3}, \lambda_{5}, \ldots\right\} / \Lambda\left\{\lambda_{1}, \lambda_{3}, \ldots, \lambda_{2 k-1}\right\}\right)_{q}$

as groups (and hence as $Z_{2}$-vector spaces). Here, $\Lambda$ is the mod 2 algebra of $A . K$. Bousfield et al. [1]; we review some of its properties in $\$ 3.1$.

In addition, we will define a map $i_{k}: B_{1}(k) \rightarrow B(k)$ and study how it relates the two spectra:

$(0.5)\left(i_{k}\right)^{*}: H^{*} B(k) \rightarrow H^{*} B_{1}(k)$ is the canonical projection.

(0.6) There exist cofibrations

$$
B_{1}(2 k+1) \stackrel{2}{\rightarrow} B_{1}(2 k+1) \stackrel{i_{2 k+1}}{\rightarrow} B(2 k+1)
$$

and

$$
B_{1}(2 k-1) \rightarrow B_{1}(2 k) \stackrel{i_{2 k}}{\rightarrow} B(2 k) .
$$

Moreover, if $M_{2}$ denotes the $Z_{2}$ Moore spectrum, then $M_{2} \wedge B_{1}(2 k) \simeq B(2 k+1)$.

$(0.7)\left(i_{k}\right)_{*}: \pi_{q}\left(B_{1}(k)\right) \rightarrow \pi_{q}(B(k))$ is injective for $1 \leqslant q \leqslant 2 k$.

As mentioned above, the Brown-Gitler spectra $B(k)$ play a prominent role in shaping the statements and proofs of the preceding results. The style of exposition will be to recite facts about $B(k)$ as they are needed.

In $\S 2$, we define the spectra $B_{1}(k)$, compute $H^{*} B_{1}(k)$, and establish the cofibrations (0.6). $\S 3$ presents the aforementioned free resolution of $H^{*} B_{1}(k)$, along with some related resolutions of $H^{*}(K(Z))=A / A\left\{\mathrm{Sq}^{1}\right\}$. Then, in $\$ 4$, we analyze the Adams spectral sequence of $B_{1}(k)$. Lastly, $\S 5$ is devoted to proving the homology surjection (0.2) and its corollary, the orientability conclusion $\left(0.2^{\prime}\right)$; the method of proof will then be used to describe various means of characterizing $B_{1}(k)$, up to homotopy 2-equivalence.

Now, $B_{1}(k)$ will be defined as a Thom spectrum over a certain filtration of the space $\Omega^{2}\left(S^{3}\langle 3\rangle\right)$, where $S^{3}\langle 3\rangle$ is the 3-connective cover of $S^{3}$. In $\S 1$, we construct a configuration space model for $\Omega^{2}\left(S^{3}\langle 3\rangle\right)$ involving the space $C_{n} X$, May's homotopy approximation to $\Omega^{n} \Sigma^{n} X$. Actually, this model, described below, arises as a corollary to a property of a certain retraction $C_{n} G \rightarrow G$, where $G$ is any abelian topological group.

$(0.8) \Omega^{2}\left(S^{3}\langle 3\rangle\right)$ is homotopy equivalent to

$$
\left\{\left\langle c_{1}, \ldots, c_{k} ; x_{1}, \ldots, x_{k}\right\rangle \in C_{2} S^{1} \mid \prod_{i=1}^{k} x_{i}=1\right\} .
$$


ACKNOWLEDGEMENTS. The material in this paper first appeared as the author's doctoral dissertation, written at Brandeis University under the direction of Edgar $\mathrm{H}$. Brown, Jr. The author would like to thank Professor Brown for his good-humored encouragement and patiently-given advice during the preparation of this work.

Also, we have learned that the spectra $B_{1}(k)$, along with certain other related spectra, have been studied extensively by Paul Goerss in his thesis [11]; many of the results given here also appear in his work.

1. An approximation to $\Omega^{2}\left(S^{3}\langle 3\rangle\right)$ and related spaces. Let $\omega: S^{n} \rightarrow K(Z, n)$ be a map representing a generator of $H^{n}\left(S^{n} ; Z\right)=Z$. Then the homotopy-theoretic fibre of $\omega$, denoted $S^{n}\langle n\rangle$, is called the $n$-connective cover of $S^{n}$.

At present, we are primarily interested in the double loop space $\Omega^{2}\left(S^{3}\langle 3\rangle\right)$. The reason is that the future sections in this paper are devoted to studying a certain family of spectra $B_{1}(k)$, and the space $\Omega^{2}\left(S^{3}\langle 3\rangle\right)$ plays an important, underlying role in the definition of these spectra. A priori, the geometry of $\Omega^{2}\left(S^{3}\langle 3\rangle\right)$ seems quite intangible. The material in this section was motivated by a desire to find a manageable geometric model for $\Omega^{2}\left(S^{3}\langle 3\rangle\right)$ along the lines of the James reduced product for $\Omega \Sigma X$ [12] or May's “little $n$-cubes monad" for $\Omega^{n} \Sigma^{n} X[\mathbf{1 6}]$.

In Theorem (1.3), we present such a model, not just for $\Omega^{2}\left(S^{3}\langle 3\rangle\right)$, but for any $\Omega^{n}\left(S^{n+1}\langle n+1\rangle\right), 1 \leqslant n<\infty$. In fact, $(1.3)$ is a consequence of a more general result involving the homotopy fibre of a certain special retraction. We should point out that the models which we end up with seem quite reasonable; unfortunately, it is not clear whether they possess the sort of combinatorial convenience that one might hope for.

The results of this section are not needed anywhere later in this work.

We begin by recounting some constructions of May. Suppose that $1 \leqslant n \leqslant \infty$. Then, given a space $X$ with base point *, define

$$
C_{n} X=\coprod_{k=1}^{\infty} F\left(R^{n}, k\right) \times_{\Sigma_{k}} X^{k} / \sim
$$

Here, $F\left(R^{n}, k\right)=\left\{\left(c_{1}, \ldots, c_{k}\right) \in\left(R^{n}\right)^{k} \mid c_{i} \neq c_{j}\right.$ if $\left.i \neq j\right\}$ is the configuration space of $k$ distinct points in the Euclidean space $R^{n}$, and $X^{k}$ is the $k$-fold Cartesian product. The symmetric group, $\Sigma_{k}$, acts on both $F\left(R^{n}, k\right)$ and $X^{k}$ by permuting coordinates. Points of $F\left(R^{n}, k\right) \times X^{k}$ will be written $\left\langle c_{1}, \ldots, c_{k} ; x_{1}, \ldots, x_{k}\right\rangle$, where $\left(c_{1}, \ldots, c_{k}\right) \in F\left(R^{n}, k\right)$ and $x_{i} \in X$. Lastly, the equivalence relation $\sim$ in $C_{n} X$ is generated by the identification

$$
\left\langle c_{1}, \ldots, c_{j}, \ldots, c_{k} ; x_{1}, \ldots, x_{j}, \ldots, x_{k}\right\rangle \sim\left\langle c_{1}, \ldots, \hat{c}_{j}, \ldots, c_{k} ; x_{1}, \ldots, \hat{x}_{j}, \ldots, x_{k}\right\rangle
$$

whenever $x_{j}=*$.

The significance of $C_{n} X$ is given by May's "approximation theorem":

Theorem (1.1) [16]. There is a map $\beta: C_{n} X \rightarrow \Omega^{n} \Sigma^{n} X$ which is a weak homotopy equivalence if $X$ is path connected. 
The key result of this chapter is the following

THEOREM (1.2). Let $G$ be an abelian topological group whose base point is the identity element 0 . Define $\mu=\mu_{n, G}: C_{n} G \rightarrow G$ by the formula

$$
\mu\left(\left\langle c_{1}, \ldots, c_{k} ; g_{1}, \ldots, g_{k}\right\rangle\right)=\sum_{i=1}^{k} g_{i} .
$$

Let $Y=Y_{n, G}$ denote the homotopy fibre of $\mu$. Then $Y \simeq \mu^{-1}(0)$. Explicitly,

$$
Y \simeq\left\{\left\langle c_{1}, \ldots, c_{k} ; g_{1}, \ldots, g_{k}\right\rangle \in C_{n} G \mid \sum_{i=1}^{k} g_{i}=0\right\} .
$$

Proof. The customary way of describing $Y$ is as the pullback of the path fibration on $G$ :

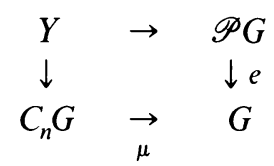

Here, $\mathscr{P} G=\{\alpha:(I, 0) \rightarrow(G, 0)\}$ is the path space of $G$ and $e(\alpha)=\alpha(1)$. Thus, we shall regard $Y$ as being

$$
\left\{\left(\left\langle c_{1}, \ldots, c_{k} ; g_{1}, \ldots, g_{k}\right\rangle, \alpha\right) \in C_{n} G \times \mathscr{P}_{G \mid \alpha}(1)=\sum_{i=1}^{k} g_{i}\right\} .
$$

Define a map $f: \mu^{-1}(0) \rightarrow Y$ by the formula

$$
f\left(\left\langle c_{1}, \ldots, c_{k} ; g_{1}, \ldots, g_{k}\right\rangle\right)=\left(\left\langle c_{1}, \ldots, c_{k} ; g_{1}, \ldots, g_{k}\right\rangle, \kappa_{0}\right),
$$

where $\kappa_{0}$ is the constant path at $0 \in G$.

We intend to show that $f$ is a homotopy equivalence by constructing an explicit homotopy inverse. However, it takes some work to set this up.

Given $0 \leqslant t \leqslant 1$, define $\phi_{t}: R \rightarrow R$ by $\phi_{t}(x)=(1-t) x+(2 t / \pi) \arctan (x)$. Note that $d\left(\phi_{t}(x)\right) / d x=1-t+2 t / \pi\left(1+x^{2}\right)>0$, so $\phi_{t}$ is one-to-one. Hence, the maps $\phi_{t}$ describe an isotopy of $R$ : observe that $\phi_{0}=\mathrm{id}_{R}$ and that $\phi_{1}$ is a homeomorphism of $R$ onto the interval $-1<x<1$.

Next, define an isotopy $\psi_{t}: R^{n} \rightarrow R^{n}$ by the equation

$$
\psi_{t}(x)= \begin{cases}\phi_{t}(|x|) x /|x| & \text { if } x \neq 0 \\ 0 & \text { if } x=0 .\end{cases}
$$

Since $\left|\psi_{t}(x)\right|=\phi_{t}(|x|), \psi_{t}$ is continuous at 0 . Again, $\psi_{t}$ is one-to-one. This time, $\psi_{0}=\operatorname{id}_{R^{n}}$ and $\psi_{1}$ gives a homeomorphism of $R^{n}$ onto $\left\{x \in R^{n}|| x \mid<1\right\}$. As $t$ goes from 0 to 1 , one can envision $\psi_{t}$ as taking points in $R^{n}$ and radially retracting them inside the unit disk.

Let $\overline{2} \in R^{n}$ denote the point $(2,0,0, \ldots, 0)$.

Finally, define $h: Y \rightarrow \mu^{-1}(0)$ by the formula

$$
h\left(\left\langle c_{1}, \ldots, c_{k} ; g_{1}, \ldots, g_{k}\right\rangle, \alpha\right)=\left\langle\psi_{1} c_{1}, \ldots, \psi_{1} c_{k}, \overline{2} ; g_{1}, \ldots, g_{k},-\sum_{i=1}^{k} g_{i}\right\rangle .
$$


The whole point is that $f$ and $h$ are homotopy inverses, and this is what we now show.

(i) $h f \simeq \mathrm{id}_{\mu^{-1}(0)}$. By tracing through the definitions, one computes that

$$
\begin{aligned}
h f\left(\left\langle c_{1}, \ldots, c_{k} ; g_{1}, \ldots, g_{k}\right\rangle\right) & =\left\langle\psi_{1} c_{1}, \ldots, \psi_{1} c_{k}, \overline{2} ; g_{1}, \ldots, g_{k},-\sum g_{i}\right\rangle \\
& \sim\left\langle\psi_{1} c_{1}, \ldots, \psi_{1} c_{k} ; g_{1}, \ldots, g_{k}\right\rangle
\end{aligned}
$$

since, by assumption, $\Sigma g_{i}=0$.

There is an evident homotopy $G_{t}: \mu^{-1}(0) \rightarrow \mu^{-1}(0)$ between $h f$ and $\operatorname{id}_{\mu^{-1}(0)}$, namely,

$$
G_{t}\left(\left\langle c_{1}, \ldots, c_{k} ; g_{1}, \ldots, g_{k}\right\rangle\right)=\left\langle\psi_{t} c_{t}, \ldots, \psi_{t} c_{k} ; g_{1}, \ldots, g_{k}\right\rangle .
$$

(ii) $f h \simeq \mathrm{id}_{Y}$. Now,

$$
f h\left(\left\langle c_{1}, \ldots, c_{k} ; g_{1}, \ldots, g_{k}\right\rangle, \alpha\right)=\left(\left\langle\psi_{1} c_{1}, \ldots, \psi_{1} c_{k}, \overline{2} ; g_{1}, \ldots, g_{k},-\sum g_{i}\right\rangle, \kappa_{0}\right) \text {. }
$$

Given $\alpha \in \mathscr{P} G$ and $0 \leqslant y \leqslant 1$, let $\alpha_{y}$, be the element of $\mathscr{P} G$ defined by $\alpha_{y}(s)=$ $\alpha(s y)$. In particular, $\alpha_{0}=\kappa_{0}$ and $\alpha_{1}=\alpha$; also, $\alpha_{y}(1)=\alpha(y)$.

Next, define a homotopy $H_{t}: Y \rightarrow Y$ by the equations

$$
\begin{aligned}
& H_{t}\left(\left\langle c_{1}, \ldots, c_{k} ; g_{1}, \ldots, g_{k}\right\rangle, \alpha\right) \\
& = \begin{cases}\left(\left\langle\psi_{2 t} c_{1}, \ldots, \psi_{2 t} c_{k} ; g_{1}, \ldots, g_{k}\right\rangle, \alpha\right) & \text { if } 0 \leqslant t \leqslant \frac{1}{2}, \\
\left(\left\langle\psi_{1} c_{1}, \ldots, \psi_{1} c_{k}, \overline{2} ; g_{1}, \ldots, g_{k}, \alpha(2-2 t)-\sum g_{i}\right\rangle, \alpha_{2-2 t}\right) & \text { if } \frac{1}{2} \leqslant t \leqslant 1 .\end{cases}
\end{aligned}
$$

Observe that $H_{t}$ is well defined when $t=\frac{1}{2}$ by applying the identification

$$
\begin{aligned}
\left(\left\langle\psi_{1} c_{1}, \ldots, \psi_{1} c_{k} ; g_{1}, \ldots, g_{k}\right\rangle, \alpha\right) & \sim\left(\left\langle\psi_{1} c_{1}, \ldots, \psi_{1} c_{k}, \overline{2} ; g_{1}, \ldots, g_{k}, 0\right\rangle, \alpha\right) \\
& =\left(\left\langle\psi_{1} c_{1}, \ldots, \psi_{1} c_{k}, \overline{2} ; g_{1}, \ldots, g_{k}, \alpha(1)-\sum g_{i}\right\rangle, \alpha_{1}\right) .
\end{aligned}
$$

Furthermore, $H_{0}=\operatorname{id}_{Y}$ and $H_{1}=f h$.

This completes the proof that $Y$ and $\mu^{-1}(0)$ are homotopy equivalent.

We shall apply (1.2) to the special case $G=S^{1}$. As usual, the group operation of $S^{1}$ will be written multiplicatively; the identity element will be denoted by 1 .

Recall the fibration $S^{n+1}\langle n+1\rangle \rightarrow S^{n+1} \rightarrow K(Z, n+1)$ described at the beginning of this section. Looping this $n$ times yields another fibration

$$
\Omega^{n}\left(S^{n+1}\langle n+1\rangle\right) \stackrel{p}{\rightarrow} \Omega^{n} S^{n+1} \stackrel{m}{\rightarrow} \Omega^{n} K(Z, n+1)=K(Z, 1)=S^{1} .
$$

The map $m$ above is of degree one in the sense that it represents a generator of $\left[\Omega^{n} S^{n+1}, S^{1}\right]=H^{1}\left(\Omega^{n} S^{n+1} ; Z\right)=Z$.

Now, by May's approximation theorem, $C_{n} S^{1}=\Omega^{n} \Sigma^{n}\left(S^{1}\right)=\Omega^{n} S^{n+1}$. In terms of the May model, the map $m: \Omega^{n} S^{n+1} \rightarrow S^{1}$ corresponds to the map $\mu_{n, S^{1}}: C_{n} S^{1} \rightarrow S^{1}$ discussed in (1.2). $\left(\mu_{n, S^{1}}\right.$ has degree one since it is split by the obvious map $x$ $\rightarrow\langle 0 ; x\rangle$.) 
Thus, there is a map of fibrations

$\begin{array}{ccc}Y_{n, S^{1}} & \stackrel{\beta^{\prime}}{\rightarrow} & \left(S^{n+1}\langle n+1\rangle\right) \\ \downarrow & \downarrow \\ C_{n} S^{1} & \stackrel{\beta}{\rightarrow} & \Omega^{n} S^{n+1} \\ \mu \downarrow & & \downarrow m \\ S^{1} & = & S^{1}\end{array}$

where $\beta$ is May's equivalence and $\beta^{\prime}$ is the induced map of homotopy fibres. By the Five Lemma, $\beta^{\prime}$ induces an isomorphism on homotopy groups. Hence, (1.2) allows us to describe the homotopy type of $\Omega^{n}\left(S^{n+1}\langle n+1\rangle\right)$ in terms of $C_{n} S^{1}$.

THEOREM (1.3). $\Omega^{n}\left(S^{n+1}\langle n+1\rangle\right)$ is homotopy equivalent to

$$
\mu^{-1}(1)=\left\{\left\langle c_{1}, \ldots, c_{k} ; x_{1}, \ldots, x_{k}\right\rangle \in C_{n} S^{1} \mid \prod_{i=1}^{h} x_{i}=1\right\} .
$$

REMARKS. (1) Of peculiar interest may be the case $n=1$. Then (1.3) describes $\Omega\left(S^{2}\langle 2\rangle\right)=\Omega S^{3}$ as $Z=\left\{x_{1} \cdots x_{k} \in J\left(S^{1}\right) \mid \prod_{i=1}^{k} x_{i}=1\right\}$, where $J\left(S^{1}\right)$ is the James reduced product of $S^{1}$. (The expression $x_{1} \cdots x_{k}$ is meant to denote a "word" of length $k$, not a multiplication in $S^{1}$.) From this point of view, the inclusion $Z \subset J\left(S^{1}\right)$ corresponds to the looped Hopf map $\Omega \eta: \Omega S^{3} \rightarrow \Omega S^{2}$.

Of course, the usual model for $\Omega S^{3}$ is the James reduced product $J\left(S^{2}\right)$.

(2) A version of (1.3) can also be formulated when $n=\infty$. Namely, let $m$ : $\Omega^{\infty} \Sigma^{\infty}\left(S^{1}\right) \rightarrow S^{1}$ be a map of degree one. Then

$$
\left\{\left\langle c_{1}, \ldots, c_{k} ; x_{1}, \ldots, x_{k}\right\rangle \in C_{x} S^{1} \mid \prod_{i=1}^{k} x_{i}=1\right\}
$$

is a model for the homotopy fibre of $m$.

2. The spectra $B_{1}(k)$-elementary properties. We now introduce the spectra $B_{1}(k)$ whose study comprises the bulk of this work. These spectra are closely related to a family of spectra $B(k)$ first constructed by Brown and Gitler in [3], and the last part of this section is devoted to establishing some simple connections between $B_{1}(k)$ and $B(k)$. For instance, thanks to a theorem of $\mathrm{R}$. Cohen, the definition of $B_{1}(k)$ leads to an obvious map $i_{k}: B_{1}(k) \rightarrow B(k)$. We shall compute $H^{*} B_{1}(k)$ as a module over the mod 2 Steenrod algebra and then show that $\left(i_{k}\right)^{*}: H^{*} B(k) \rightarrow H^{*} B_{1}(k)$ is a certain natural projection. (It amounts to killing $\mathrm{Sq}^{1}$.) In addition, we will show that $M_{2} \wedge B_{1}(2 k) \simeq B(2 k+1)$, where $M_{2}$ is the $Z_{2}$ Moore spectrum. This will allow us to prove the existence of a cofibration of spectra $B_{1}(k-1) \rightarrow B_{1}(k) \stackrel{i_{k}}{\rightarrow} B(k)$. As a result, $B_{1}(k)$ can be realized as a cofibre; that is, there is a map $f: \Sigma^{-1} B(k) \rightarrow$ $B_{1}(k-1)$ such that $B_{1}(k) \simeq B_{1}(k-1) \cup{ }_{f} C\left(\Sigma^{-1} B(k)\right)$.

The spectra $B_{1}(k)$ are defined in $\S 2.2$; up until then, we record some necessary background information. 
2.1. Preliminaries. All homology and cohomology should be taken with coefficients in $Z_{2}$, the integers mod 2, unless otherwise noted.

Let $A$ denote the mod 2 Steenrod algebra, and let $\chi: A \rightarrow A$ be the canonical antiautomorphism. In what follows, we will of ten be concerned with studying certain cyclic modules over $A$, as described by

DEFINITION (2.1). Given $k \geqslant 0$, let

(a) $M(k)=A / A\left\{\chi\left(\mathrm{Sq}^{i}\right) \mid i>k\right\}$; and

(b) $M_{1}(k)=A / A\left\{\mathrm{Sq}^{1}, \chi\left(\mathrm{Sq}^{i}\right) \mid i>k\right\}$.

Remarks. (1) One can also write $M_{1}(k)$ as $M(k) \otimes_{A_{0}} Z_{2}$, where $A_{0}$ is the exterior subalgebra of $A$ generated by $\mathrm{Sq}^{1}$ and where the right action of $A_{0}$ on $M(k)$ is induced by right multiplication.

(2) Since $\chi\left(\mathrm{Sq}^{2 k+1}\right)=\chi\left(\mathrm{Sq}^{1} \mathrm{Sq}^{2 k}\right)=\chi\left(\mathrm{Sq}^{2 k}\right) \mathrm{Sq}^{1}$, one easily sees that $M_{1}(2 k)=$ $M_{1}(2 k+1)$. Indeed, many of the definitions that we propose will contain this type of redundancy. However, we will continue to allow for both odd and even cases, as it will help make certain results (e.g., (2.15), (4.9)) easier to state.

The additive structures of $M(k)$ and $M_{1}(k)$ are not hard to determine:

THEOREM (2.2). (a) $M(k)$ has an additive basis given by $\left\{\chi\left(\mathrm{Sq}^{I}\right) \mid \mathrm{Sq}^{I}\right.$ is admissible, $I=\left(i_{1}, \ldots, i_{t}\right)$, and $\left.i_{1} \leqslant k\right\}$.

(b) $M_{\mathrm{i}}(k)$ has an additive basis given by $\left\{\chi\left(\mathrm{Sq}^{I}\right) \mid \mathrm{Sq}^{I}\right.$ is admissible, $I=\left(i_{1}, \ldots, i_{t}\right)$, $i_{1} \leqslant k$, and $\left.i_{1}=0(\bmod 2)\right\}$.

Proof. (a) is a straightforward consequence of the Adem relations.

To prove (b), note first that if $i_{1}=1(\bmod 2)$, then

$$
\chi\left(\mathrm{Sq}^{I}\right)=\chi\left(\mathrm{Sq}^{1} \mathrm{Sq}^{i_{1}-1} \mathrm{Sq}^{i_{2}} \ldots \mathrm{Sq}^{i_{t}}\right)=\chi\left(\mathrm{Sq}^{i_{1}-1} \mathrm{Sq}^{i_{2}} \cdots \mathrm{Sq}^{i_{t}}\right) \mathrm{Sq}^{1}=0
$$

in $M_{1}(k)$. To see that the elements described in (b) are linearly independent, observe that $\chi\left(\mathrm{Sq}^{2 i+1}\right) \mathrm{Sq}^{1}=\chi\left(\mathrm{Sq}^{1} \mathrm{Sq}^{2 i+1}\right)=0$, so that, in order to have $\chi\left(\mathrm{Sq}^{I}\right)$ in $A\left\{\mathrm{Sq}^{1}\right\}$, $i_{1}$ must be odd. This, along with the Adem relations, implies (b).

A good reason for studying the module $M(k)$ is supplied by the work of Brown and Gitler. They constructed a spectrum $B(k)$, now known as the "Brown-Gitler spectrum," one of whose properties is

TheOREM (2.3) [3]. $H^{*}(B(k))=M(k)$ as A-modules.

$M(k)$ also made a rather unexpected appearance in certain constructions of Mahowald, which we now review.

Let $h: S^{1} \rightarrow B O$ represent the generator of $\pi_{1} B O=Z_{2} . h$ admits a canonical extension $\Omega^{2} \Sigma^{2} h: \Omega^{2} \Sigma^{2} S^{1}=\Omega^{2} S^{3} \rightarrow \Omega^{2} \Sigma^{2} B O$, and, since $B O$ is an infinite loop space, there is a retraction $r: \Omega^{2} \Sigma^{2} B O \rightarrow B O$. Let $\gamma: \Omega^{2} S^{3} \rightarrow B O$ be the composite

$$
\Omega^{2} S^{3} \stackrel{\Omega^{2} \Sigma^{2} h}{\rightarrow} \Omega^{2} \Sigma^{2} B O \stackrel{r}{\rightarrow} B O .
$$

Mahowald [14, Corollary 4.5] made the striking observation that $T(\gamma) \simeq K\left(Z_{2}\right)$, where $T(\gamma)$ is the Thom spectrum associated to $\gamma$, and $K\left(Z_{2}\right)$ is the $Z_{2}$ EilenbergMac Lane spectrum, both normalized to have bottom homology class in dimension zero. 
Let $C_{2} S^{1}$ be May's configuration space model for $\Omega^{2} S^{3}$, as described in $\S 1 . C_{2} S^{1}$ has an obvious filtration, namely, in the notation of $\S 1$,

$$
F_{j}\left(C_{2} S^{1}\right)=\coprod_{k=1}^{j} F\left(R^{2}, k\right) \times_{\Sigma_{k}}\left(S^{1}\right)^{k} / \sim .
$$

By May's approximation theorem, $C_{2} S^{1} \simeq \Omega^{2} S^{3}$, and hence we can also regard $\Omega^{2} S^{3}$ as a filtered space. Abbreviate the $j$ th stage of this filtration to $F_{j}$, so that $\Omega^{2} S^{3}=\bigcup F_{j}, *=F_{0} \subset F_{1} \subset F_{2} \subset \ldots$

Let $\gamma_{k}=\left.\gamma\right|_{F_{k}}$, i.e., $\gamma_{k}$ is the composite $F_{k} \subset \Omega^{2} S^{3} \stackrel{\gamma}{\rightarrow} B O$. The possibility of realizing the Brown-Gitler spectrum $B(k)$ as the Thom spectrum $T\left(\gamma_{k}\right)$ was suggested by a calculation of Mahowald and then concluded by a proof of R. Cohen.

TheOREM (2.4) (MAHOWALD [14]). $H^{*}\left(T\left(\gamma_{k}\right)\right)=M(k)$ as A-modules.

THEOREM (2.5) (R. COHEN [9]). $T\left(\gamma_{k}\right)$ is homotopy 2-equivalent to $B(k)$.

(We should point out that, due to the manner in which it is constructed, $B(k)$ is trivial at odd primes.)

REMARKs. (1) For our purposes, two spectra will be called homotopy 2-equivalent if their 2-completions are homotopy equivalent. A suitable reference on completions is Bousfield and Kan [2, especially Chapters I, VI]. The 2-completion we shall use is what Bousfield and Kan refer to as the " $Z_{2}$-completion."

(2) In [14], Mahowald also studied the filtered quotients $F_{k} / F_{k-1}$ of the May decomposition of $\Omega^{2} S^{3}$. It is easy to see that $F_{k} / F_{k-1}$ is the Thom space of the $k$-plane bundle

$$
F\left(R^{2}, k\right) \times_{\Sigma_{k}}\left(R^{1}\right)^{k} \rightarrow F\left(R^{2}, k\right) \times_{\Sigma_{k}}(\text { pt. }) .
$$

Mahowald showed that $H^{*}\left(F_{k} / F_{k-1}\right)=\Sigma^{k} M([k / 2])$. Brown and Peterson [6] then followed up on this by proving that $F_{k} / F_{k-1}$ gives another realization of the Brown-Gitler spectrum, i.e., $F_{k} / F_{k-1}$ is homotopy 2-equivalent to $\Sigma^{k} B([k / 2])$.

Let $x_{1} \in H_{1}\left(\Omega^{2} S^{3}\right)$ be the generator and set $x_{i}=\left(Q_{1}\right)^{i-1}\left(x_{1}\right)$ in the usual lower-index Dyer-Lashof notation. Given a monomial $m=x_{1}^{\alpha_{1}} \cdots x_{n}^{\alpha_{n}}$, define the weight of $m$, wt $(m)$, to be $\sum_{j=1}^{n} \alpha_{j} 2^{j-1}$. The following theorem contains well-known results of May and F. Cohen.

THEOREM (2.6) [7, I, III]. (a) $H_{*}\left(\Omega^{2} S^{3}\right)=Z_{2}\left[x_{1}, x_{2}, \ldots\right]$, where $x_{i} \in H_{2^{i}-1}\left(\Omega^{2} S^{3}\right)$. (b) $H_{*}\left(F_{k}\right)$ has a $Z_{2}$-basis consisting of all monomials $m$ such that $\operatorname{wt}(m) \leqslant k$.

Now recall the fibration $\Omega^{2} S^{3}\langle 3\rangle \stackrel{p}{\rightarrow} \Omega^{2} S^{3} \stackrel{m}{\rightarrow} S^{1}$ of $\S 1$, where $S^{3}\langle 3\rangle$ is the 3-connective cover of $S^{3}$. If $\alpha: S^{1} \rightarrow \Omega^{2} S^{3}$ represents a generator of $\pi_{1}\left(\Omega^{2} S^{3}\right)=Z$, then it is easy to check that $(\alpha+p): S^{1} \times \Omega^{2} S^{3}\langle 3\rangle \rightarrow \Omega^{2} S^{3}$ induces an isomorphism on homotopy groups and hence is a homotopy equivalence. ("+" above denotes the loop sum in $\Omega^{2} S^{3}$.) Thus, $H_{*}\left(\Omega^{2} S^{3}\right)=H_{*} S^{1} \otimes H_{*}\left(\Omega^{2} S^{3}\langle 3\rangle\right)$. Moreover, the map $p: \Omega^{2} S^{3}\langle 3\rangle \rightarrow \Omega^{2} S^{3}$ is a double loop map so that

$$
p_{*}: H_{*}\left(\Omega^{2} S^{3}\langle 3\rangle\right) \rightarrow H_{*}\left(\Omega^{2} S^{3}\right)
$$


is a ring homomorphism which commutes with $Q_{1}$. In light of (2.6), this establishes

TheOREM (2.7). $H_{*}\left(\Omega^{2} S^{3}\langle 3\rangle\right)=Z_{2}\left[x_{1}^{2}, x_{2}, x_{3} \ldots\right] \subset H_{*}\left(\Omega^{2} S^{3}\right)$.

Note that the set of all monomials of even weight forms an additive basis for $H_{*}\left(\Omega^{2} S^{3}\langle 3\rangle\right)$.

One outgrowth of (2.6) is that $H_{*}\left(\Omega^{2} S^{3}\right)$ and the Steenrod algebra $A$ are isomorphic as graded vector spaces; the link between them is provided by Milnor's analysis of the dual of the Steenrod algebra [17]. Milnor's isomorphism will come in handy later, and so now we briefly recall how it is defined.

Let $\mathscr{M}$ be the set of all sequences of nonnegative integers having finitely many nonzero entries: $J=\left(\alpha_{1}, \ldots, \alpha_{n}, 0,0, \ldots\right)$. Let $\mathscr{N}$ be the set of all sequences $I$ such that $\mathrm{Sq}^{I}$ is admissible in $A$. Define $\theta: \mathscr{M} \rightarrow \mathscr{N}$ by $\theta\left(\alpha_{1}, \ldots, \alpha_{n}, 0,0, \ldots\right)=\left(i_{1}, \ldots, i_{n}\right)$, where $i_{q}=\sum_{j=q}^{n} 2^{j-q} \alpha_{j}$; for instance, $i_{1}=\alpha_{1}+2 \alpha_{2}+4 \alpha_{3}+\cdots+2^{n}{ }^{1} \alpha_{n}$.

Lemma (2.8). $\theta: \mathscr{M} \rightarrow \mathcal{N}$ is a bijection of sets.

Proof. $\theta$ has an inverse $\phi: \mathscr{N} \rightarrow \mathscr{M}$ defined by

$$
\phi\left(i_{1}, \ldots, i_{n}\right)=\left(i_{1}-2 i_{2}, \ldots, i_{n} 1-2 i_{n}, i_{n}, 0,0, \ldots\right) .
$$

Next, given a monomial $m=x_{1}^{\alpha_{1}} \cdots x_{n}^{\alpha_{n}}$, define $\theta_{*}(m)=\chi\left(\mathrm{Sq}^{\theta\left(\alpha_{1} \ldots \ldots \alpha_{n}(0,0) \ldots\right)}\right)$. Extending this by $Z_{2}$-linearity, one obtains a vector space homomorphism $\theta_{*}$ : $H_{*}\left(\Omega^{2} S^{3}\right) \rightarrow A$.

LemMa (2.9). (a) $\theta_{*}$ is an isomorphism of graded vector spaces.

(b) If $\theta_{*}(m)=\chi\left(\mathrm{Sq}^{I}\right)$ as above and $I=\left(i_{1}, \ldots, i_{n}\right)$, then $i_{1}=\mathrm{wt}(m)$.

Proof. Checking that $\theta_{*}$ preserves graded dimension is a triviality. Also, (2.8) implies that $\theta$ induces a one-to-one correspondence between basis elements, and this proves (a). (b) follows directly from the definition of $\theta$.

We now return to the study of Thom spectra.

Let $g: \Omega^{2} S^{3}\langle 3\rangle \rightarrow B O$ denote the composition $\Omega^{2} S^{3}\langle 3\rangle \stackrel{p}{\rightarrow} \Omega^{2} S^{3} \stackrel{\gamma}{\rightarrow} B O$. Mahowald [15] recognized that the Thom spectrum $T(g)$ was another familiar object:

THEOREM (2.10). $T(g)$ is homotopy 2-equivalent to the Eilenberg-Mac Lane spectrum $K(Z)$.

Proof. Since $H^{1}\left(\Omega^{2} S^{3}\langle 3\rangle\right)=0$, the map $g$ lifts to $\tilde{g}: \Omega^{2} S^{3}\langle 3\rangle \rightarrow B S O$, i.e., $T(g)$ has an integral Thom class. Let $U: T(g) \rightarrow K(Z)$ represent this class. We shall prove that $U$ is a 2-equivalence by showing that it induces an isomorphism in mod 2 cohomology.

The map $p: \Omega^{2} S^{3}\langle 3\rangle \rightarrow \Omega^{2} S^{3}$ induces a map on the level of Thom spectra $T(p)$ : $T(g) \rightarrow T(\gamma) \simeq K\left(Z_{2}\right)$ which is nontrivial; thus, $T(p)$ represents the mod 2 Thom class. This gives a commutative diagram

$$
\begin{array}{ccc} 
& & K(Z) \\
& U \nearrow & \downarrow r \\
& \underset{T(p)}{\rightarrow} & K\left(Z_{2}\right)
\end{array}
$$


where $r$ represents reduction mod 2. Hence, there is a diagram in cohomology:

$$
\begin{array}{ccc} 
& & H^{*}(K(Z))=A / A\left\{\mathrm{Sq}^{1}\right\} \\
& H^{*}(T(g)) \underset{U^{*} \swarrow}{\leftarrow} & H^{*}\left(K\left(Z_{2}\right)\right)=A
\end{array}
$$

Now, $p_{*}: H_{*}\left(\Omega^{2} S^{3}\langle 3\rangle\right) \rightarrow H_{*}\left(\Omega^{2} S^{3}\right)$ is injective, so, after applying the Thom isomorphism, $T(p)^{*}$ must be surjective in cohomology. This in turn implies that $U^{*}$ : $A / A\left\{\mathrm{Sq}^{1}\right\} \rightarrow H^{*}(T(g))$ is surjective. Thus, to prove that $U^{*}$ is an isomorphism, it suffices to show that $A / A\left\{\mathrm{Sq}^{1}\right\}$ and $H^{*}(T(g))$ have the same rank over $Z_{2}$ in each graded dimension

By the Thom isomorphism, $H^{*}(T(g))=H_{*}\left(\Omega^{2} S^{3}\langle 3\rangle\right)$ as graded vector spaces. Recall that $H_{*}\left(\Omega^{2} S^{3}\langle 3\rangle\right)$ has a basis consisting of monomials of even weight. On the other hand, by (2.2)(b) when $k=\infty, A / A\left\{\mathrm{Sq}^{1}\right\}$ has a basis consisting of $\chi\left(\mathrm{Sq}^{\left(i_{1}, \ldots, i_{n}\right)}\right)$ with $i_{1}$ even. A one-to-one dimension-preserving correspondence between these basis elements is provided by (2.9), and this completes the proof.

The idea of using (2.8) in conjunction with the Thom isomorphism for proofs of this type seems to be due to F. Cohen, May and Taylor [8, p. 105].

2.2. The spectra $B_{1}(k)$. We now commence the study of the spectra $B_{1}(k)$. The definition to be given was originally presented by Mahowald [15, p. 554]. (See the appendix for an alternative, non-Thom spectrum definition.)

In view of the homotopy equivalence $\Omega^{2} S^{3} \simeq S^{1} \times \Omega^{2} S^{3}\langle 3\rangle$, Mahowald allows the May filtration on $\Omega^{2} S^{3}$ to induce a filtration on $\Omega^{2} S^{3}\langle 3\rangle$. Let $W_{2 n} \subset \Omega^{2} S^{3}\langle 3\rangle$ denote the stage of this induced filtration such that $W_{2 n} \subset F_{2 n}$ and $H_{*} W_{2 n}=H_{*} F_{2 n}$ $\cap H_{*}\left(\Omega^{2} S^{3}\langle 3\rangle\right)$. Also, define $W_{2 n+1}$ to be equal to $W_{2 n}$. In this case, by (2.6)(b) and (2.7), the preceding homological equation is still true, i.e., $H_{*} W_{2 n+1}=H_{*} F_{2 n+1} \cap$ $H_{*}\left(\Omega^{2} S^{3}\langle 3\rangle\right)$. Either way, $H_{*} W_{k}$ has an additive basis comprised of those monomials $m$ in the $x_{i}$ which satisfy $\operatorname{wt}(m) \leqslant k$ and $\operatorname{wt}(m)=0(\bmod 2)$.

Note that, for any $k$, there are "canonical" inclusions $W_{k} \subset F_{k}$, namely, either $W_{2 n} \subset F_{2 n}$ or $W_{2 n+1}=W_{2 n} \subset F_{2 n} \subset F_{2 n+1}$.

Finally, let $g_{k}: W_{k} \rightarrow B O$ denote the composite

$$
W_{k} \subset F_{k} \stackrel{\gamma_{k}}{\rightarrow} B O .
$$

Definition (2.11). The spectrum $B_{1}(k)$ is defined to be the Thom spectrum $T\left(g_{k}\right)$, completed at 2 .

REMARKS. (1) $B_{1}(2 k)=B_{1}(2 k+1)$. (The definition was not meant to disguise this.)

(2) The spectra $B_{1}(k)$ played an essential role in Mahowald's presentation of $b o$-resolutions in [13], as well as in a subsequent elaboration of that exposition by Davis, Gitler and Mahowald [10].

Induced over the inclusion $W_{k} \subset F_{k}$ is a map of Thom spectra $T\left(g_{k}\right) \rightarrow T\left(\gamma_{k}\right)$. Completing this at 2 and then using R. Cohen's Theorem (2.5), one obtains a map $i_{k}: B_{1}(k) \rightarrow B(k)$. 
In view of the relationship between $B(k)$ and $K\left(Z_{2}\right)$ as Thom spectra, one can look at the realization of $K(Z)$ in $(2.10)$ and then begin to regard $B_{1}(k)$ as being what an integral version of the Brown-Gitler spectrum should look like, at least as far as 2-primary information is concerned. Such an interpretation is enhanced by

THEOREM (2.12). $H^{*}\left(B_{1}(k)\right)=M_{1}(k)$ as A-modules. Furthermore, $\left(i_{k}\right)^{*}$ : $H^{*}(B(k)) \rightarrow H^{*}\left(B_{1}(k)\right)$ is the obvious projection $\pi: M(k) \rightarrow M_{1}(k)$.

Proof. The arguments here are similar in spirit to those given in the proof of (2.10).

The inclusion $W_{k} \subset F_{k}$ induces a homomorphism $H_{*} W_{k} \rightarrow H_{*} F_{k}$ which is injective. Thus, by dualizing and translating to the Thom spectrum level via the Thom isomorphism, we conclude that $\left(i_{k}\right)^{*}: H^{*} B(k) \rightarrow H^{*} B_{1}(k)$ is surjective. Moreover,

$$
\begin{aligned}
H^{1} B_{1}(k) & =H^{1} W_{k} \quad(\text { by the Thom isomorphism) } \\
& =\left(H_{1} W_{k}\right)^{*}=0,
\end{aligned}
$$

so that $\left(i_{k}\right)^{*} \mathrm{Sq}^{1}=0$. This implies that $\left(i_{k}\right)^{*}$ factors

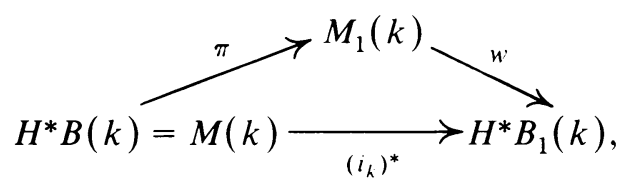

where $w$ must also be surjective.

As shown in (2.2)(b), $M_{1}(k)$ has a $Z_{2}$-basis given by those $\chi\left(\mathrm{Sq}^{I}\right)$ with $i_{1} \leqslant k$ and $i_{1}=0(\bmod 2)$. On the other hand, $H^{*} B_{1}(k)=H_{*} W_{k}$ as vector spaces by the Thom isomorphism, and we know that $H_{*} W_{k}$ has a basis given by monomials $m$ with $\operatorname{wt}(m) \leqslant k$ and $\operatorname{wt}(m)=0(\bmod 2)$. Thus, (2.9) shows that $M_{1}(k)$ and $H^{*} B_{1}(k)$ have the same rank over $\mathbf{Z}_{2}$ in each graded dimension, and this implies that $w$ must be an isomorphism.

The assertion in the theorem concerning $\left(i_{k}\right)^{*}$ is forced by the fact that $\left(i_{k}\right)^{*}$ is $A$-linear.

The next lemma is easily verified.

Lemma (2.13). The following sequence of $A$-modules is exact:

$$
0 \rightarrow M_{1}(k-1) \stackrel{\beta}{\rightarrow} M(k) \stackrel{\pi}{\rightarrow} M_{1}(k) \rightarrow 0,
$$

where $\beta(1)=\mathrm{Sq}^{1}$ and $\pi(1)=1$.

Proof. Use the description of the bases for these modules given in (2.2).

We shall show that this exact sequence can be realized by a cofibration. To do this, let $M_{2}$ denote the stable $Z_{2}$ Moore complex $S^{0} \cup_{2} e^{1}$.

LeMma (2.14). $M_{2} \wedge B_{1}(2 k) \simeq B(2 k+1)$.

Proof. This fact seems first to have been noticed by Mahowald [15].

Consider the composition $c: S^{1} \times W_{2 k} \subset F_{1} \times F_{2 k} \rightarrow F_{2 k+1}$, the last map coming from the multiplicative properties of the May filtration of $\Omega^{2} S^{3}$ [16]. It is easy to check that $c$ induces an isomorphism in homology. 
Next, there is a commutative diagram

$$
\begin{array}{lll}
S^{1} \times W_{2 k} & \stackrel{c}{\rightarrow} & F_{2 k+1} \\
h+g_{2 k} \searrow & & \swarrow \gamma_{2 k+1} \\
& \text { BO } &
\end{array}
$$

where $h: S^{1} \rightarrow B O$ represents the nontrivial element of $\pi_{1} B O$. The Thom spectrum $T(h)$ is the Moore spectrum $M_{2}$. Thus, $c$ induces a map $T(c): M_{2} \wedge B_{1}(2 k) \rightarrow$ $B(2 k+1)$ which is an isomorphism in homology and, consequently, must be an equivalence.

THEOREM (2.15). There is a cofibration

$$
B_{1}(k-1) \rightarrow B_{1}(k) \stackrel{i_{k}}{\rightarrow} B(k)
$$

whose long exact sequence in cohomology realizes (2.13).

Proof. Case 1. $k=2 n+1$. Let $S^{0} \stackrel{2}{\rightarrow} S^{0} \stackrel{j}{\rightarrow} M_{2}$ be the usual cofibration. Since cofibrations are preserved by smash products, one obtains a cofibration

$$
S^{0} \wedge B_{1}(2 n) \stackrel{2 \wedge \text { id }}{\rightarrow} S^{0} \wedge B_{1}(2 n) \stackrel{j \wedge \text { id }}{\rightarrow} M_{2} \wedge B_{1}(2 n),
$$

which can be rewritten

$$
B_{1}(2 n) \stackrel{2}{\rightarrow} B_{1}(2 n+1) \stackrel{j \wedge \text { id }}{\rightarrow} B(2 n+1)
$$

using (2.14). (Recall that $B_{1}(2 n)=B_{1}(2 n+1)$.) Tracing through the arguments given in (2.14), it is easy to check that $j \wedge$ id is the same as the standard map $i_{2 n+1}$, as desired.

Case 2. $k=2 n$. Let $X$ denote the cofibre of $i_{2 n}: B_{1}(2 n) \rightarrow B(2 n)$. Since $\left(i_{2 n}\right)^{*}$ is surjective, the long exact sequence in cohomology of $B_{1}(2 n) \stackrel{i_{2 n}}{\rightarrow} B(2 n) \rightarrow X$ breaks up into a series of short exact sequences

$$
0 \rightarrow H^{q} X \rightarrow H^{q} B(2 n) \stackrel{\left(i_{2 n}\right)^{*}}{\rightarrow} H^{q} B_{1}(2 n) \rightarrow 0 .
$$

Thus, by (2.13), $H^{*} X=\Sigma M_{1}(2 n-1)$.

Over the obvious maps of base spaces

$$
\begin{array}{ccc}
W_{2 n-1} & \subset & F_{2 n-1} \\
\cap & & \cap \\
W_{2 n} & \subset & F_{2 n}
\end{array}
$$

there are maps of Thom spectra:

$$
\begin{array}{ccccc}
B_{1}(2 n-1) & \stackrel{i_{2 n-1}}{\rightarrow} & B(2 n-1) & \rightarrow & \sum B_{1}(2 n-2) \\
\downarrow & & \downarrow \zeta & & \downarrow \bar{\xi} \\
B_{1}(2 n) & \stackrel{i_{2 n}}{\rightarrow} & B(2 n) & \rightarrow & X
\end{array}
$$


Here, $\bar{\zeta}$ is the induced map of cofibres, the cofibre of $i_{2 n-1}$ having been identified as $\Sigma B_{1}(2 n-2)$ in Case 1 . We will prove that $\bar{\zeta}$ is an equivalence by showing that it induces an isomorphism in cohomology.

Since $H^{*} X=\Sigma M_{1}(2 n-1)=\Sigma M_{1}(2 n-2)=H^{*} \Sigma B_{1}(2 n-2)$ and since these modules are cyclic over $A$, it suffices by $A$-linearity to check that $\bar{\zeta}^{*}: H^{1} X \rightarrow$ $H^{1} \Sigma B_{1}(2 n-2)$ is an isomorphism, i.e., that $\bar{\zeta}^{*}(1)=1$. But, by using the Thom isomorphism, it is easy to see that $\zeta^{*}: H^{1} B(2 n) \rightarrow H^{1} B(2 n-1)$ satisfies $\zeta^{*}\left(\mathrm{Sq}^{1}\right)=$ $\mathrm{Sq}^{1}$. The desired result follows from the exact sequences (2.13).

The upshot of all this is that $X \simeq \Sigma B_{1}(2 n-2)=\Sigma B_{1}(2 n-1)$, so that there is a cofibration

$$
B_{1}(2 n) \stackrel{i_{2 n} n}{\rightarrow} B(2 n) \rightarrow \Sigma B_{1}(2 n-1) .
$$

This completes the proof.

REMARKS. (1) The proof of Case 1 shows that, when $k$ is odd, the cofibration of (2.15) should really be written

$$
B_{1}(2 n+1) \stackrel{2}{\rightarrow} B_{1}(2 n+1) \rightarrow B(2 n+1) .
$$

(2) One implication of (2.15) is that the Brown-Gitler spectrum $B(k)$ can be realized as a cofibre. Namely, there is a map $g: B_{1}(k-1) \rightarrow B_{1}(k)$ of degree 2 on the bottom cell such that $B(k) \simeq B_{1}(k) \cup_{g} C\left(B_{1}(k-1)\right.$ ).

Brown and Peterson [6] obtained $B(k)$ as a cofibre in a different way. Specifically, they proved that there exist cofibrations of the form $\Sigma^{k-1} B([k / 2]) \stackrel{h}{\rightarrow} B(k-1) \rightarrow$ $B(k)$ and hence

$$
B(k) \simeq B(k-1) \cup{ }_{h} C\left(\Sigma^{k-1} B([k / 2])\right) .
$$

3. Some families of subcomplexes of the $\Lambda$-algebra. In this section, we shall construct explicit free resolutions of the $A$-modules $M_{1}(k)$. These will be used later in the Adams spectral sequence and Postnikov analyses of $B_{1}(k)$. In addition, we will describe a family of closely related resolutions of $A / A\left\{\mathrm{Sq}^{1}\right\}$ which are needed in some of the naturality arguments in $\S 5$.

3.1. A resolution of $M_{1}(k)$. Each of the resolutions to be discussed in this section will be a subcomplex of a certain fixed $A$-resolution of $Z_{2}$, the so-called " $\Lambda$-algebra" resolution. To recall, let $\bar{\Lambda}$ be the free associative $Z_{2}$-algebra with unit generated by $\lambda_{i}, i=-1,0,1, \ldots$, modulo the relations

$$
\lambda_{i} \lambda_{j}=\sum\left(\begin{array}{c}
s-1 \\
2 s-(j-2 i)
\end{array}\right) \lambda_{i+s} \lambda_{j-s} \quad \text { whenever } 2 i<j .
$$

Then let $\Lambda=\bar{\Lambda} / \bar{\Lambda}\left\{\lambda_{-1}\right\} ; \Lambda$ is the algebra introduced by Bousfield et al. in [1].

Grade $\Lambda$ by setting $\operatorname{dim} \lambda_{i}=i$.

If $I=\left(i_{1}, i_{2}, \ldots, i_{q}\right)$, let $\lambda_{I}=\lambda_{i_{1}} \lambda_{i_{2}} \cdots \lambda_{i_{q}}$. Also, define $l(I)=q$ and $t(I)=i_{q}$. The sequence $I$ is called admissible if $t(I) \geqslant 0$ and $2 i_{j} \geqslant i_{j+1}$ for $j=1,2, \ldots, q-1$. (By convention, if $I=()$, then $\lambda_{I}=1, l(I)=0, t(I)=\infty$, and $I$ is considered admissible.) In [1], it is shown that

Lemma (3.2). $\left\{\lambda_{I} \mid I\right.$ admissible $\}$ is an additive basis for $\Lambda$. 
Next, let $\Lambda^{*}=\operatorname{Hom}_{Z_{2}}\left(\Lambda, Z_{2}\right)$ be the graded vector space dual and let $\left\{\lambda^{I}\right\}$ denote the dual basis with respect to the basis of (3.2). On the free $A$-module $A \otimes \Lambda^{*}$, there is an $A$-linear differential $d: A \otimes \Lambda^{*} \rightarrow A \otimes \Lambda^{*}$ which acts on basis elements according to the formula

$$
d\left(1 \otimes \lambda^{I}\right)=\sum \lambda^{I}\left(\lambda_{j} \lambda_{J}\right) \chi\left(\mathrm{Sq}^{j+1}\right) \otimes \lambda^{J} .
$$

The summation on the right runs over all $j \geqslant-1$ and all basis elements $\lambda^{J}$. From now on, given $a \in A$ and $\lambda \in \Lambda^{*}$, the symbol $a \otimes \lambda$ will be abbreviated to $a \lambda$.

LEMmA (3.4). If $\lambda^{I}\left(\lambda_{j} \lambda_{J}\right) \neq 0$, then $t(J) \geqslant t(I)$ and $l(J)=l(I)-1$.

Proof. This follows by induction on $l(I)$, using the relations (3.1).

For the remainder of this section, let $k \geqslant 0$ be a fixed integer.

We now recall the Brown-Gitler resolution of $M(k)=A / A\left\{\chi\left(\mathrm{Sq}^{i}\right) \mid i>k\right\}$. Let

$$
D_{q}\left(=D_{q}(k)\right)=A \otimes\left\{\lambda^{I} \mid I \text { admissible, } l(I)=q, t(I) \geqslant k\right\},
$$

viewed as an $A$-submodule of $A \otimes \Lambda^{*}$. In particular, $D_{0}=A \otimes\left\{\lambda^{()}\right\}=A$. From (3.4), it follows immediately that $d\left(D_{q}\right) \subset D_{q-1}$. Brown and Gitler proved

THEOREM (3.5) [3]. The sequence

$$
\cdots \rightarrow D_{q} \stackrel{d}{\rightarrow} D_{q-1} \rightarrow \cdots \rightarrow D_{0} \stackrel{\varepsilon}{\rightarrow} M(k) \rightarrow 0
$$

is exact, where $\varepsilon\left(\lambda^{()}\right)=1$.

Note that $M(0)=Z_{2}$ so that, when $k=0,(3.5)$ gives an $A$-free resolution of $Z_{2}$. The resolution we will construct for $M_{1}(k)$ lies between this full $\Lambda$-algebra resolution of $Z_{2}$ and the Brown-Gitler resolution of $M(k)$.

Given sequences $I=\left(i_{1}, \ldots, i_{q}\right)$ and $J=\left(j_{1}, \ldots, j_{r}\right)$, let $(I, J)=$ $\left(i_{1}, \ldots, i_{q}, j_{1}, \ldots, j_{r}\right)$ and $\lambda^{I} \lambda^{J}=\lambda^{(I, J)}$.

Definition (3.6). The sequence $I$ is called $k$-acceptable if $I=\left(I^{\prime}, Z\right)$ where $l\left(I^{\prime}\right) \geqslant 0, t\left(I^{\prime}\right) \geqslant k-1$ if $k$ is even, $t\left(I^{\prime}\right) \geqslant k$ if $k$ is odd, $Z=(0, \ldots, 0)$, and $l(Z) \geqslant 1$.

Now define certain free $A$-modules $C_{q}=C_{q}(k)$ as follows:

$$
\begin{aligned}
C_{q}=A \otimes\left\{\lambda^{I} \mid I \text { admissible, } l(I)=q,\right. \text { and } \\
\text { either } t(I) \geqslant k \text { or } I \text { is } k \text {-acceptable }\} .
\end{aligned}
$$

We view $C_{q}$ as a submodule of $A \otimes \Lambda^{*}$. In terms of the Brown-Gitler resolution above,

$$
C_{q}(k)=D_{q}(k) \oplus\left(A \otimes\left\{\lambda^{I} \mid I \text { admissible, } l(I)=q, I k \text {-acceptable }\right\}\right) ;
$$

in particular, $D_{q}(k) \subset C_{q}(k)$. Also, $C_{0}=D_{0}=A \otimes\left\{\lambda^{()}\right\}=A$.

The main point of this section is that the $C_{q}$ 's, together with the differential $d$ above, form a resolution of $M_{1}(k)$. This will be proven in Theorem (3.10); first, however, some calculations are required.

Lemma (3.8). Suppose that $I=\left(i_{1}, \ldots, i_{q}\right)$. Then

$$
d \lambda^{(I, 0)}=\left(d \lambda^{I}\right) \lambda^{0}+i_{q} \lambda^{\left(i_{1} \ldots, i_{q} \cdot i_{i}+1\right)} .
$$


Proof. Write

$$
d \lambda^{(I, 0)}=\sum_{t(J)>0} \lambda^{(I, 0)}\left(\lambda_{j} \lambda_{J}\right) \chi\left(\mathrm{Sq}^{j+1}\right) \lambda^{J}+\sum_{t(J)=0} \lambda^{(I, 0)}\left(\lambda_{j} \lambda_{J}\right) \chi\left(\mathrm{Sq}^{j+1}\right) \lambda^{J} .
$$

Suppose that $t(J)>0$. By the relations (3.1), if $2 i<m$, then $\lambda_{i} \lambda_{m}=\sum \lambda_{I_{\alpha}}$, where each $I_{\alpha}$ is admissible and $t\left(I_{\alpha}\right) \geqslant 2 i+1$. Thus, in order to get $\lambda^{(I .0)}\left(\lambda_{j} \lambda_{J}\right) \neq 0, j$ must equal -1 . Let $J=\left(j_{1}, \ldots, j_{q}\right), j_{q}>0$. Once again using (3.1),

$$
\lambda_{-1} \lambda_{J}=\lambda_{j_{1}} \lambda_{-1} \lambda_{j_{2}} \cdots \lambda_{j_{q}}+\left(\sum \lambda_{J_{\alpha}}\right) \lambda_{j_{2}} \cdots \lambda_{j_{q}}
$$

where $t\left(J_{\alpha}\right) \geqslant 0$. The second term can be expanded in terms of admissible monomials $\lambda_{T_{\gamma}}$ with $t\left(T_{\gamma}\right) \geqslant 1$. Consequently,

$$
\lambda^{(I, 0)}\left(\lambda_{-1} \lambda_{J}\right)=\lambda^{(I, 0)}\left(\lambda_{j_{1}} \lambda_{-1} \lambda_{j_{2}} \cdots \lambda_{j_{4}}\right)
$$

Repeating this argument, it follows that

$$
\lambda^{(I, 0)}\left(\lambda_{-1} \lambda_{J}\right)=\lambda^{(I, 0)}\left(\lambda_{j_{1}} \lambda_{j_{2}} \cdots \lambda_{j_{q-1}} \lambda_{-1} \lambda_{j_{q}}\right)
$$

Now, $\lambda_{-1} \lambda_{j_{q}}=\left(j_{q}-1\right) \lambda_{j_{q}-1} \lambda_{0}+\sum \lambda_{U_{\alpha}}$, where $t\left(U_{\alpha}\right) \geqslant 1$. Thus,

$$
\lambda^{(I, 0)}\left(\lambda_{-1} \lambda_{J}\right)=\left(j_{q}-1\right) \lambda^{(I, 0)}\left(\lambda_{j_{1}} \cdots \lambda_{j_{4}} \lambda_{j_{4}-1} \lambda_{0}\right) \text {. }
$$

This will be nonzero if and only if $J=\left(i_{1}, \ldots, i_{q-1}, i_{q}+1\right)$ and $i_{q}=1(\bmod 2)$. Hence,

$$
\sum_{t(J)>0} \lambda^{(I, 0)}\left(\lambda_{j} \lambda_{J}\right) \chi\left(\mathrm{Sq}^{j+1}\right) \lambda^{J}=i_{q} \lambda^{\left(i_{1}, i_{2}, \ldots, i_{4-1}, i_{q}+1\right)}
$$

Next, assume that $t(J)=0$, that is, $J=\left(J^{\prime}, 0\right)$. Observe that if $\lambda_{j} \lambda_{J^{\prime}}=\sum \lambda_{J_{\alpha}}$, where each $J_{\alpha}$ is admissible, then $\lambda_{j} \lambda_{\left(J^{\prime}, 0\right)}=\sum \lambda_{\left(J_{\alpha}, 0\right)}$, where each $\left(J_{\alpha}, 0\right)$ is admissible. Thus, $\lambda^{(I, 0)}\left(\lambda_{j} \lambda_{\left(J^{\prime}, 0\right)}\right) \neq 0$ if and only if $\lambda^{I}\left(\lambda_{j} \lambda_{J^{\prime}}\right) \neq 0$. As a result,

$$
\sum_{t(J)=0} \lambda^{(I, 0)}\left(\lambda_{j} \lambda_{J}\right) \chi\left(\mathrm{Sq}^{j+1}\right) \lambda^{J}=\sum \lambda^{I}\left(\lambda_{j} \lambda_{J^{\prime}}\right) \chi\left(\mathrm{Sq}^{j+1}\right) \lambda^{\left(J^{\prime}, 0\right)}=\left(d \lambda^{I}\right) \lambda^{0}
$$

Combining the preceding two paragraphs, we obtain (3.8).

Lemma (3.9). $d\left(C_{q}\right) \subset C_{q-1}$.

Proof. It suffices to show that $d \lambda^{I} \in C_{q-1}$ for each basis element $\lambda^{I}$ in $C_{q}$. For those $\lambda^{I}$ with $t(I) \geqslant k$, this follows from (3.4). For those $\lambda^{I}$ with $I k$-acceptable, it follows by iterating (3.8) and then applying (3.4).

We are now prepared to prove that the $C_{q}$ 's give a free resolution of $M_{1}(k)$.

THEOREM (3.10). The sequence

$$
\cdots \rightarrow C_{q} \stackrel{d}{\rightarrow} C_{q-1} \rightarrow \cdots \rightarrow C_{0} \stackrel{\varepsilon}{\rightarrow} M_{1}(k) \rightarrow 0
$$

is exact, where $\varepsilon\left(\lambda^{()}\right)=1$.

Proof. The following arguments are modelled quite closely on Brown and Gitler's proof of (3.5).

Exactness at $C_{0}: d \lambda^{i}=\chi\left(\mathrm{Sq}^{i+1}\right) \lambda^{()}$, and $\left\{\lambda^{i} \mid i \geqslant k\right.$ or $\left.i=0\right\}$ is a basis for $C_{1}$.

Exactness at $C_{q}, q>0$ : This resolution is a subcomplex of the full $\Lambda$-algebra resolution, so $d^{2}=0$. 
Suppose that $u \in C_{q}$ and that $u \in \operatorname{ker} d$. We need to show that $u=d v$ for some $v \in C_{q+1}$.

Order all sequences of length $q$ lexicographically from the right, that is, if $I=\left(i_{1}, \ldots, i_{q}\right)$ and $J=\left(j_{1}, \ldots, j_{q}\right)$, then $I>J$ if there exists $t \geqslant 1$ such that $i_{t}>j_{t}$ and $i_{s}=j_{s}$ for $s>t$. Observe that $(0, \ldots, 0)$, the sequence of $q$ zeros, is the minimum element.

Given any inadmissible sequence $K$, one can use (3.1) and induction on $l(K)$ to show that $\lambda_{K}=\sum \lambda_{K_{\alpha}}$, where each $K_{\alpha}$ is admissible and $K_{\alpha}<K$. It then follows from the definition of $d,(3.3)$, that, if $I=(i, J)$ is admissible, then

$$
d \lambda^{I}=\chi\left(\mathrm{Sq}^{i+1}\right) \lambda^{J}+\sum_{J^{\prime}>J} b_{J^{\prime}} \lambda^{J^{\prime}}
$$

Next, express $u=\sum a_{\alpha} \lambda^{I_{\alpha}}, a_{\alpha} \neq 0$, where each $I_{\alpha}$ is admissible, and let $I_{0}=\min I_{\alpha}$ with respect to the above ordering.

Case 1. $I_{0}>(0, \ldots, 0)$. (Thus, $I_{0} \geqslant(k-1,0, \ldots, 0)$ if $k$ is even and $I_{0} \geqslant$ $(k, 0, \ldots, 0)$ if $k$ is odd.) Write $I_{0}=(i, J)$. Then $u=\sum_{j \geqslant i} a_{j} \lambda^{(j . J)}+\sum c_{I^{\prime}} \lambda^{I^{\prime}}$, where $I^{\prime}=\left(i^{\prime}, J^{\prime}\right)$ and $J^{\prime}>J$. Hence, by (3.11),

$$
0=d u=\sum_{j \geqslant i} a_{j} \chi\left(\mathrm{Sq}^{j+1}\right) \lambda^{J}+\sum_{J^{\prime}>J} b_{J^{\prime}} \lambda^{J^{\prime}}
$$

In particular, $\sum_{j \geqslant i} a_{j} \chi\left(\mathrm{Sq}^{j+1}\right)=0$. Suppose that $a_{i}=\sum \chi\left(\mathrm{Sq}^{I_{t}}\right)$, where each $L_{t}$ is admissible in the usual Steenrod algebra sense. Then

$$
\begin{aligned}
0 & =\sum_{t} \chi\left(\mathrm{Sq}^{I_{t}}\right) \chi\left(\mathrm{Sq}^{i+1}\right)+\sum_{j>i} a_{j} \chi\left(\mathrm{Sq}^{j+1}\right) \\
& =\sum_{t} \chi\left(\mathrm{Sq}^{\left(i+1, L_{t}\right)}\right)+\sum_{j>i} \chi\left(\mathrm{Sq}^{j+1} a_{j}^{\prime}\right),
\end{aligned}
$$

where $a_{j}^{\prime}=\chi\left(a_{j}\right)$. According to the Adem relations, if $\mathrm{Sq}^{B}$ is admissible, then $\mathrm{Sq}^{(a \cdot B)}$ is either admissible or can be written as a sum of $\mathrm{Sq}^{B_{j}}$ 's where the first entry of $B_{j}$ is greater than $a$. Consequently, after expressing each $a_{j}^{\prime}$ in terms of admissible monomials, one sees that, in the preceding sum, the $\left(i+1, L_{t}\right)$ cannot all be admissible. Say that $\left(i+1, L_{0}\right)$ is not, so that $L_{0}=(j+1, K)$, where $i \leqslant 2 j$. Note that

$$
d \lambda^{(j, i, J)}=d \lambda^{\left(j, I_{0}\right)}=\chi\left(\mathrm{Sq}^{j+1}\right) \lambda^{I_{0}}+\sum_{T>I_{0}} c_{T} \lambda^{T}
$$

so adding $d\left(\chi\left(\mathrm{Sq}^{K}\right) \lambda^{\left(j . I_{0}\right)}\right)$ to $u$ replaces $\chi\left(\mathrm{Sq}^{L_{0}}\right) \lambda^{I_{0}}$ by terms involving $\lambda^{T}, T>I_{0}$. Moreover, $\lambda^{\left(j, I_{0}\right)} \in C_{q+1}$. (Note the use of $I_{0}>(0, \ldots, 0)$ here.) Continuing in this way, we may express $u=d v+$ terms in $\lambda^{T}$ with $T$ arbitrarily large. But $\operatorname{dim} \lambda^{T}$ increases with $T$. Hence, $u=d v$, as desired.

Case 2. $I_{0}=(0, \ldots, 0)$. We will find $w \in C_{q}$ such that $w=u+d v$ for some $v \in C_{q+1}$ and such that $w$ satisfies the conditions for Case 1.

Let $u=a_{0} \lambda^{I_{0}}+\sum_{T>I_{0}} a_{T} \lambda^{T}$.

Since $\chi\left(\mathrm{Sq}^{2 i+1}\right)=\chi\left(\mathrm{Sq}^{1} \mathrm{Sq}^{2 i}\right)=\chi\left(\mathrm{Sq}^{2 i}\right) \mathrm{Sq}^{1}$, we may write $a_{0}=b \mathrm{Sq}^{1}+$ $\sum \chi\left(\mathrm{Sq}^{\left(m_{t}, J_{t}\right)}\right)$, where each $\left(m_{t}, J_{t}\right)$ is admissible and $m_{t}=0(\bmod 2)$. Now, by $(3.8)$, 


$$
\begin{aligned}
& d \lambda^{(0 \ldots \ldots)}=\mathrm{Sq}^{1} \lambda^{(0 \ldots \ldots 0)} \text {. Let } u_{1}=u+d\left(b \lambda^{(0 \ldots \ldots)}\right) \text {. Then } \\
& u_{1}=\sum \chi\left(\mathrm{Sq}^{\left(m_{t}, J_{t}\right)}\right) \lambda^{\left.I_{0}\right)}+\sum_{T>I_{0}} a_{T} \lambda^{T} .
\end{aligned}
$$

Also,

$$
\begin{aligned}
0 & =d u_{1}=\sum \chi\left(\mathrm{Sq}^{\left(m_{t}, J_{t}\right)}\right) \mathrm{Sq}^{1} \lambda^{(0 \ldots \ldots 0)}+\sum_{T>I_{0}} a_{T} d \lambda^{T} \\
& =\sum \chi\left(\mathrm{Sq}^{\left(m_{t}+1 . J_{t}\right)}\right) \lambda^{(0 \ldots \ldots 0)}+\sum_{T>I_{0}} a_{T} d \lambda^{T} .
\end{aligned}
$$

Each of the sequences $\left(m_{t}+1, J_{t}\right)$ is admissible, and hence each $\chi\left(\mathrm{Sq}^{\left(m_{t}+1, J_{t}\right)}\right) \lambda^{(0 \ldots \ldots)}$ must be cancelled by a term coming from some $a_{T} d \lambda^{T}$. Choose a particular $\left(m_{t}+1, J_{t}\right)$ and suppose that $\chi\left(\mathrm{Sq}^{\left(m_{t}+1, J_{t}\right)}\right) \lambda^{(0 \ldots \ldots)}$ is cancelled by a term coming from $a_{T} d \lambda^{T}$. According to (3.11), in order to contribute to such a cancellation, $T$ must be of the form $(j, 0, \ldots, 0)$ :

$$
\begin{aligned}
d\left(a_{T} \lambda^{(j, 0 \ldots, 0)}\right) & =a_{T} \chi\left(\mathrm{Sq}^{j+1}\right) \lambda^{(0, \ldots 0)}+\sum_{K>(0, \ldots .0)} c_{K} \lambda^{K} \\
& =\chi\left(\mathrm{Sq}^{j+1} a_{T}^{\prime}\right) \lambda^{(0, \ldots 0)}+\sum_{K>(0, \ldots .0)} c_{K} \lambda^{K},
\end{aligned}
$$

where $a_{T}^{\prime}=\chi\left(a_{T}\right)$. Using the Adem relations, one can see that $j+1 \leqslant m_{t}+1$, that is, $j \leqslant m_{t}$. But, by definition of $C_{q}$,

$$
j \geqslant \begin{cases}k-1 & \text { if } k \text { is even, } \\ k & \text { if } k \text { is odd. }\end{cases}
$$

Since $m_{t}$ is even, this implies that

$$
m_{t} \geqslant \begin{cases}k & \text { if } k \text { is even, } \\ k+1 & \text { if } k \text { is odd }\end{cases}
$$

Therefore, $\lambda^{\left(m_{t}-1.0, \ldots 0\right)} \in C_{q+1}$.

Now,

$$
\begin{aligned}
d\left(\sum \chi\left(\mathrm{Sq}^{J_{t}}\right) \lambda^{\left(m_{t}-1,0 \ldots \ldots\right)}\right) & =\sum \chi\left(\mathrm{Sq}^{J_{t}}\right) \chi\left(\mathrm{Sq}^{m_{t}}\right) \lambda^{(0 \ldots \ldots 0)}+\sum_{K>(0 \ldots \ldots)} e_{K} \lambda^{K} \\
& =\sum \chi\left(\mathrm{Sq}^{\left(m_{t} J_{t}\right)}\right) \lambda^{I_{0}}+\sum_{K>I_{0}} e_{K} \lambda^{K} .
\end{aligned}
$$

Let

$$
\begin{aligned}
w & =u_{1}+d\left(\sum \chi\left(\mathrm{Sq}^{J_{t}}\right) \lambda^{\left(m_{t}-1.0 \ldots \ldots 0\right)}\right) \\
& =u+d\left(b \lambda^{(0, \ldots 0)}+\sum \chi\left(\mathrm{Sq}^{J_{t}}\right) \lambda^{\left(m_{t}-1.0 \ldots .0\right)}\right) .
\end{aligned}
$$

Then $w=\sum_{T>(0, \ldots, 0)} x_{T} \lambda^{T}$, which reduces the problem to Case 1, thereby completing the proof.

3.2. Some resolutions of $A / A\left\{\mathrm{Sq}^{1}\right\}$. As mentioned earlier, the modules $C_{q}$ of (3.7) can be decomposed into two pieces, one piece being the modules $D_{q}$ that Brown and Gitler use for $M(k)$ and the other piece involving the " $k$-acceptable" basis elements. Inasmuch as one passes from $M(k)$ to $M_{1}(k)$ by killing $\mathrm{Sq}^{1}$, the $k$-acceptable piece 
can be regarded as accounting for the relation $\mathrm{Sq}^{1}=0$ and other higher order relations associated to it. In $\$ 5$, we shall need to use this idea. More precisely, we shall require $A$-free resolutions of $A / A\left\{\mathrm{Sq}^{1}\right\}$ which include the $k$-acceptable basis elements; the remainder of this section is devoted to constructing such resolutions.

Define certain free $A$-modules $E_{q}=E_{q}(k)$ as follows:

$$
\begin{array}{r}
E_{q}=E_{q}(k)=A \otimes\left\{\lambda^{I} \mid I \text { admissible, } l(I)=q, \text { and either }(\mathrm{i}) t(I) \geqslant k\right. \\
\text { and } t(I)=0(\bmod 2) \text { or (ii) } I \text { is } k \text {-acceptable }\} .
\end{array}
$$

As usual, we regard $E_{q}$ as a submodule of $A \otimes \Lambda^{*}$. Note that $E_{q} \subset C_{q}$ and that $C_{q}=D_{q}+E_{q}$ (though not a direct sum). Also, $\left.E_{0}=C_{0}=A \otimes\left\{\lambda^{(}\right)\right\}=A$. In Theorem (3.15), we will prove that the $E_{q}$ 's with the differential $d$ form a resolution of $A / A\left\{\mathrm{Sq}^{1}\right\}$, but first there are some preliminaries.

LEMMA (3.13). Suppose that $I$ and $J$ are admissible sequences such that $t(I)=0$ $(\bmod 2)$ and $t(J)=1(\bmod 2)$. Then $\lambda^{I}\left(\lambda_{j} \lambda_{J}\right)=0$.

Proof. It follows from (3.1) and standard arguments involving $\bmod 2$ binomial coefficients (e.g., [18, Lemma 2.6]) that, if $J^{\prime}$ is inadmissible and $t\left(J^{\prime}\right)=1(\bmod 2)$, then $\lambda_{J^{\prime}}=\sum \lambda_{J_{\alpha}}$, where $J_{\alpha}$ is admissible and $t\left(J_{\alpha}\right)=1(\bmod 2)$. The lemma is an immediate consequence.

REMARK. Let $\Lambda_{\text {even }}^{*}$ be the subspace of $\Lambda^{*}$ with basis $\left\{\lambda^{I} \mid I\right.$ admissible, $t(I)=0$ $(\bmod 2)\}$. The content of the lemma is that $d\left(A \otimes \Lambda_{\text {even }}^{*}\right) \subset A \otimes \Lambda_{\text {even }}^{*}$.

LEMMA (3.14). $d\left(E_{q}\right) \subset E_{q-1}$.

Proof. It suffices to show that $d \lambda^{I} \in E_{q-1}$ for each basis element $\lambda^{I}$ in $E_{q}$. For those $\lambda^{I}$ with $t(I) \geqslant k$ and $t(I)=0(\bmod 2)$, this follows from $(3.4)$ and $(3.13)$. For those $\lambda^{I}$ with $I k$-acceptable, it follows by iterating (3.8) and then applying (3.4).

THEOREM (3.15). For each $k$, the sequence

$$
\cdots \rightarrow E_{q} \stackrel{d}{\rightarrow} E_{q-1} \rightarrow \cdots \rightarrow E_{0} \stackrel{\varepsilon}{\rightarrow} A / A\left\{\mathrm{Sq}^{1}\right\} \rightarrow 0
$$

is exact, where $\varepsilon\left(\lambda^{()}\right)=1$.

Proof. Exactness at $E_{0}$ : Note that

$$
E_{1}=A \otimes\left\{\lambda^{i} \mid i=0 \text { or } i=2 j \geqslant k\right\} \text {. }
$$

For $\lambda^{i}$ of this type, $d \lambda^{i}=\chi\left(\mathrm{Sq}^{i+1}\right) \lambda^{()}=\chi\left(\mathrm{Sq}^{1} \mathrm{Sq}^{i}\right) \lambda^{()}=\chi\left(\mathrm{Sq}^{i}\right) \mathrm{Sq}^{1} \lambda^{()}$, and hence $d\left(E_{1}\right)=\left(A\left\{\mathrm{Sq}^{1}\right\}\right) \lambda^{()}$.

Exactness of $E_{q}, q>0$ : The arguments are identical to those used in the proof of (3.10).

RemarKs. (1) A series of closely related resolutions of $A / A\left\{\mathrm{Sq}^{1}\right\}$ can be constructed by tacking a fixed number of zeros onto the ends of all the basis elements of the $E_{q}$ 's. That is, let $Z(t)=(0, \ldots, 0)$ be the sequence consisting of $t$ zeros, and let $E_{q} \lambda^{Z(t)}=A \otimes\left\{\lambda^{(I, Z(t))} \mid \lambda^{I} \in E_{q}\right\}$. Then the arguments that went into the proof of 
(3.15) can easily be extended to show that the following subcomplex of the $\Lambda$-algebra resolution is also exact:

$$
\begin{gathered}
\cdots \rightarrow E_{q} \lambda^{Z(t)} \rightarrow E_{q-1} \lambda^{Z(t)} \rightarrow \cdots \rightarrow E_{0} \lambda^{Z(t)} \rightarrow A \otimes\left\{\lambda^{Z(t-1)}\right\} \rightarrow \\
\cdots \rightarrow A \otimes\left\{\lambda^{0}\right\} \rightarrow A \otimes\left\{\lambda^{()}\right\} \rightarrow A / A\left\{\mathrm{Sq}^{1}\right\} \rightarrow 0 .
\end{gathered}
$$

(2) Observe that (3.13) implies that the differential $d$ induces a map $d^{\prime}: A \otimes$ $\left(\Lambda^{*} / \Lambda_{\text {even }}^{*}\right) \rightarrow A \otimes\left(\Lambda^{*} / \Lambda_{\text {even }}^{*}\right)$. (See the remark following (3.13).) We have learned that Paul Goerss has used this idea to obtain resolutions of the modules $M_{1}(k)$ in the following manner [11].

One can think of $\left\{\lambda^{\prime} \mid I\right.$ admissible, $\left.t(I)=1(\bmod 2)\right\}$ as an additive basis for $\Lambda^{*} / \Lambda_{\text {even }}^{*}$. Define

$$
\begin{array}{r}
C_{q}^{\prime}\left(=C_{q}^{\prime}(k)\right)=A \otimes\left\{\lambda^{I} \mid I \text { admissible, } l(I)=q, t(I) \geqslant k,\right. \\
\text { and } t(I)=1(\bmod 2)\},
\end{array}
$$

regarded as a submodule of $A \otimes\left(\Lambda^{*} / \Lambda_{\text {even }}^{*}\right)$. Then Goerss shows that the sequence

$$
\cdots \rightarrow C_{q}^{\prime} \stackrel{d^{\prime}}{\rightarrow} C_{q-1}^{\prime} \rightarrow \cdots \rightarrow C_{1}^{\prime} \rightarrow A / A\left\{\mathrm{Sq}^{1}\right\} \rightarrow M_{1}(k) \rightarrow 0
$$

is exact.

4. On the homotopy groups of $B_{1}(k)$. In this section, we use the Adams spectral sequence to compute the homotopy groups of $B_{1}(k)$ up through a dimension roughly equal to $2 k$. By calculating in the $\Lambda$-algebra via the resolution of $H^{*} B_{1}(k)=M_{1}(k)$ constructed in $\S 3$, we obtain the $E_{2}$ term. Then, to pass to $E_{\propto}$ and solve the subsequent extensions, we translate the problem to the Brown-Gitler spectrum $B(k)$, where the corresponding problems have already been solved.

More precisely, the following results are obtained:

(a) $\pi_{0}\left(B_{1}(k)\right)=Z_{\hat{2}}\left(Z_{\hat{2}}=\lim Z_{2^{\prime}}\right.$, the 2-adic integers).

(b) All elements of $\pi_{q}\left(B_{1}(2 \overleftarrow{k})\right)=\pi_{q}\left(B_{1}(2 k+1)\right), 1 \leqslant q \leqslant 4 k+2$, are of order 2 . A $Z_{2}$-basis for these groups is given by

$\left\{\lambda_{I} \mid I\right.$ admissible, $\operatorname{dim} \lambda_{I} \leqslant 4 k+2, t(I) \geqslant 2 k+1$, and $\left.t(I)=1(\bmod 2)\right\}$.

(c) $\left(i_{k}\right)_{*}: \pi_{q}\left(B_{1}(k)\right) \rightarrow \pi_{q}(B(k))$ is injective for $1 \leqslant q \leqslant 2 k$.

To begin, given a spectrum $X,\left\{\left(E_{r}(X), d_{r}\right)\right\}$ will denote the Adams spectral sequence of $X$. The bigrading of $E_{r}(X)$ is the usual one so that a nonzero element $x \in E_{r}^{s, t}(X)$ which survives to $E_{\infty}^{s, t}(X)$ contributes to an extension in $\pi_{t-s}(X) . t-s$ is called the total dimension of $x$.

As explained in $\S 3$, the free $A$-module $A \otimes \Lambda^{*}$ has a differential $d$ which gives rise to an $A$-free resolution of $H^{*} S^{0}=Z_{2}$, where $S^{0}$ is the sphere spectrum. (This is Theorem (3.5) for $k=0$.) Thus, $\left(E_{1}\left(S^{0}\right), d_{1}\right)=\left(\operatorname{Hom}_{A}\left(A \otimes \Lambda^{*}, Z_{2}\right), d^{*}\right)$. Now, the primary appeal of using the $\Lambda$-algebra is that this $E_{1}$ term is tractable [1]; namely, for this resolution, $\left(E_{1}\left(S^{0}\right), d_{1}\right)=(\Lambda, \partial)$, where $\partial: \Lambda \rightarrow \Lambda$ gives $\Lambda$ the structure of a graded differential algebra and satisfies

$$
\partial \lambda_{i}=\sum\left(\begin{array}{c}
s-1 \\
2 s-i-2
\end{array}\right) \lambda_{s-1} \lambda_{i-s} .
$$


The action of $\partial$ is identical to multiplication on the left by $\lambda_{-1}$. We remark that, as an element of $E_{1}\left(S^{0}\right), \lambda_{I}$ has total dimension equal to the dimension of $\lambda_{I}$ in $\Lambda$.

We next summarize some of the Adams spectral sequence properties of the Brown-Gitler spectrum $B(k)$. Let $\mathscr{D}=\mathscr{D}(k)$ denote the resolution of $H^{*} B(k)=$ $M(k)$ described in Theorem (3.5):

$$
\mathscr{D}: \cdots \rightarrow D_{q} \stackrel{d}{\rightarrow} D_{q-1} \rightarrow \cdots \rightarrow D_{0} \stackrel{\varepsilon}{\rightarrow} M(k) \rightarrow 0 .
$$

Recall that $D_{q}=A \otimes\left\{\lambda^{I} \mid I\right.$ admissible, $\left.l(I)=q, t(I) \geqslant k\right\}$. For this resolution, $E_{1}(B(k))=\operatorname{Hom}_{A}\left(\mathscr{D}, Z_{2}\right)$. Since $\mathscr{D}$ is a subcomplex of $A \otimes \Lambda^{*}, E_{1}(B(k))$ can be thought of as a quotient of $\Lambda$; specifically, $E_{1}(B(k))=\Lambda / \Lambda\left\{\lambda_{0}, \ldots, \lambda_{k-1}\right\}$. From this point of view, $E_{1}(B(k))$ has a $Z_{2}$-basis consisting of those $\lambda_{I}$ with $I$ admissible and $t(I) \geqslant k$, and $d_{1}: E_{1}(B(k)) \rightarrow E_{1}(B(k))$ can be computed by using $\partial$, followed by passing to the quotient.

The following theorem is a reasonably straightforward consequence of Brown and Gitler's construction of $B(k)$ in [3]; details have since been written up by Brown and Peterson.

THEOREM (4.2) [4, THEOREM 5.1]. (a) $E_{\infty}^{s, t}(B(k))=E_{1}^{s, t}(B(k))$ provided that $t-s$ $\leqslant 2 k$ (i.e., the spectral sequence collapses in this range).

(b) All elements of $\pi_{q}(B(k)), q<2 k$, are of order 2 (i.e., there are no nontrivial extensions in this range).

REMARK. Observe that, by using (4.2)(a), one can compute the order of $\pi_{2 k}(B(k))$, at least in principle; however, the extensions have not been completely determined. It is known that elements of order greater than 2 do exist. For instance, Brown and Peterson [4] have used a map $f \in \pi_{2^{j+1}}\left(B\left(2^{j}\right)\right)$ of order $2^{j+2}$ which is represented by $\lambda_{2^{j+1}}$ in order to shorten Mahowald's construction of a nontrivial element $\eta_{j+2} \in$ $\pi_{2^{j+2}}\left(S^{0}\right)[14]$.

We now begin to analyze the Adams spectral sequence of the spectrum $B_{1}(k)$. Let $\mathscr{C}=\mathscr{C}(k)$ denote the resolution of $H^{*} B_{1}(k)=M_{1}(k)$ given in Theorem (3.10):

$$
\mathscr{C}: \cdots \rightarrow C_{q} \stackrel{d}{\rightarrow} C_{q-1} \rightarrow \cdots \rightarrow C_{0} \stackrel{\varepsilon}{\rightarrow} M_{1}(k) \rightarrow 0 .
$$

Recall that $C_{q}=A \otimes\left\{\lambda^{I} \mid I\right.$ admissible, $l(I)=q$, and either $t(I) \geqslant k$ or $I$ is $k$ acceptable $\}$. Like $\mathscr{D}$, the resolution $\mathscr{C}$ is a subcomplex of $A \otimes \Lambda^{*}$. As a result, $E_{1}\left(B_{1}(k)\right)=\operatorname{Hom}_{A}\left(\mathscr{C}, Z_{2}\right)$ can be regarded as a quotient of $\Lambda$ and $d_{1}: E_{1}\left(B_{1}(k)\right)$ $\rightarrow E_{1}\left(B_{1}(k)\right)$ is the $Z_{2}$-quotient map induced by $\partial$. In this setting,

$\left\{\lambda_{I} \mid I\right.$ admissible and either $t(I) \geqslant k$ or $I$ is $k$-acceptable $\}$ is a $Z_{2}$-basis for $E_{1}\left(B_{1}(k)\right)$.

The following lemma was proven by Brown and Gitler in [3] and in fact is implicit in the statement of (4.2)(a).

Lemma (4.3). Suppose that $\operatorname{dim} \lambda_{I} \leqslant 2 k+1$. Then $\partial \lambda_{I} \in \Lambda\left\{\lambda_{0}, \ldots, \lambda_{k-1}\right\}$.

Proof. As defined in $\S 3, \bar{\Lambda}$ is the free associative $Z_{2}$-algebra generated by $\lambda_{i}$, $i=-1,0,1, \ldots$, modulo the relations (3.1), and $\Lambda=\bar{\Lambda} / \bar{\Lambda}\left\{\lambda_{-1}\right\}$. Let $J_{n} \subset \bar{\Lambda}$ be the left ideal $\bar{\Lambda}\left\{\lambda_{-1}, \lambda_{0}, \ldots, \lambda_{n-1}\right\}$. 
We will show by induction on $l(I)$ that

$$
J_{n} \lambda_{I} \subset J_{s},
$$

where $s=\left[\left(2 n+\operatorname{dim} \lambda_{I}\right) / 2\right]$. Since, in $\Lambda, \partial \lambda_{I}=\lambda_{-1} \lambda_{l}$, the lemma will follow from (4.4) by setting $n=0$.

Suppose $l(I)=1$, i.e., $I=(i)$. Let $j<n$ be given. If $2 j \geqslant i$, then $\lambda_{j} \lambda_{i} \in J_{i+1} \subset$ $J_{[(2 n+i) / 2]}$. On the other hand, if $2 j<i$, then $\lambda_{j} \lambda_{i} \in J_{[(2 n+i) / 2]}$ by (3.1). Next, suppose that $I=\left(i, I^{\prime}\right), l\left(I^{\prime}\right)>0$, and that (4.4) is true for $I^{\prime}$. Then

$$
J_{n} \lambda_{I}=\left(J_{n} \lambda_{i}\right) \lambda_{I^{\prime}} \subset J_{[(2 n+i) / 2]} \lambda_{I^{\prime}} \subset J_{s} .
$$

This proves (4.4) and, as mentioned, implies the lemma.

Lemma (4.5). Suppose that $I=\left(i_{1}, \ldots, i_{q}\right)$ is admissible, $i_{q}>0$. Then $\partial \lambda_{I}=$ $\left(i_{q}-1\right) \lambda_{\left(i_{1} \ldots i_{q}, i_{q}-1,0\right)}+\sum \lambda_{I_{a}}$, where $I_{\alpha}$ is admissible and $t\left(I_{\alpha}\right) \geqslant 1$.

Proof. This calculation was carried out as part of the proof of (3.8).

A rather technical computation now yields $E_{2}\left(B_{1}(k)\right)$ in total dimension less than or equal to $2 k$, as follows.

LEMma (4.6). For $t-s \leqslant 2 k, E_{2}^{s . t}\left(B_{1}(k)\right)$ has a $Z_{2}$-basis consisting of $\lambda_{(0, \ldots(0)}=\lambda_{0}^{j}$, $j \geqslant 0$, and $\lambda_{I}$, where $I$ is admissible, $t(I) \geqslant k$, and $t(I)=1(\bmod 2)$.

Proof. The result is trivial if $k=0$, so assume $k>0$.

As remarked earlier, if $\partial \lambda_{I}=\sum \varepsilon_{\alpha} \lambda_{I_{u}}$, where each $I_{\alpha}$ is admissible, then, in $E_{1}\left(B_{1}(k)\right), d_{1} \lambda_{I}=\sum \varepsilon_{\alpha} \lambda_{I_{a}}$, where this latter sum runs only over those $\lambda_{I_{\alpha}}$ in $E_{1}\left(B_{1}(k)\right)$. This procedure will now be used to identity cycles and boundaries of $E_{1}\left(B_{1}(k)\right)$ in the desired range of dimensions.

For the rest of this proof, all sequences $I$ should be assumed admissible with $\operatorname{dim} \lambda_{I} \leqslant 2 k+1$. Let $Z(t)=(0, \ldots, 0)$, the sequence of $t$ zeros.

Suppose that $I=\left(i_{1}, \ldots, i_{q}\right), i_{q}>0$. By (4.3) and (4.5), we may write

$$
\partial \lambda_{I}=\left(i_{q}-1\right) \lambda_{\left(i_{1} \ldots i_{q}, i_{q} \cdots 1,()\right)}+\sum \lambda_{\left(I_{\alpha}, k-1\right)}+\sum \lambda_{J_{\beta}},
$$

where $0<t\left(J_{\beta}\right)<k-1$. Moreover, since $\partial \lambda_{0}=0$ and $\partial$ is a derivation,

$$
\begin{aligned}
\partial \lambda_{(I, Z(t))}= & \left(\partial \lambda_{l}\right) \lambda_{Z(t)}=\left(i_{q}-1\right) \lambda_{\left(i_{1} \ldots i_{q}, i_{4}-1, Z(t+1)\right)} \\
& +\sum \lambda_{\left(I_{\alpha}, k-1, Z(t)\right)}+\sum \lambda_{\left(J_{\beta}, Z(t)\right)} .
\end{aligned}
$$

This yields the following formulas in $E_{1}\left(B_{1}(k)\right)$ :

(4.7a) If $t(I)=i_{q} \geqslant k$, then $d_{1} \lambda_{I}=\left(i_{q}-1\right) \lambda_{\left(i_{1} \ldots . i_{q}, i_{q}-1.0\right)}$.

(4.7b) If $(I, Z(t))$ is $k$-acceptable, $l(I) \geqslant 1$, and $t(I)=i_{q}>0$, then

$$
d_{1} \lambda_{(I . Z(t))}=\left(i_{q}-1\right) \lambda_{\left(i_{1} \ldots i_{q}, i_{q}-1, Z(t+1)\right)}+(k-1) \sum \lambda_{\left(I_{\alpha}, k-1 . Z(t)\right)} \cdot
$$

A careful inspection of these formulas (see the following note) reveals that, for $t-s \leqslant 2 k$, the $E_{1}\left(B_{1}(k)\right)$-cycles have a basis consisting of those $\lambda_{l}$ for which either:

(i) $I=Z(t), t \geqslant 0$;

(ii) $t(I) \geqslant k, t(I)=1(\bmod 2)$; or

(iii) $I=\left(I^{\prime}, Z(t)\right)$ is $k$-acceptable, $t\left(I^{\prime}\right)=1(\bmod 2)$; 
and that the $E_{1}\left(B_{1}(k)\right)$-boundaries are generated by precisely those elements of type (iii). This produces the $E_{2}$ term stated in the lemma.

(Note on proof. When $k$ is even, the analysis given above requires some special care because sequences of the form $I=\left(I^{\prime}, k-1, Z(t)\right)$ are then $k$-acceptable. This accounts for the presence of the second term on the right-hand side of formula (4.7b). However, one can show by induction on $t$ that, for sequences $I$ of this form, $\lambda_{I}$ is a boundary, using (4.7a) to start the induction and (4.7b) to continue. It then follows directly from (4.7b) that all basis elements of type (iii) are boundaries; in fact, this is probably the easiest way to see that such elements are cycles. Clearly, (4.7a) and (4.7b) imply that type (iii) elements generate all possible boundaries, and it is not hard to check that (i)-(iii) account for all possible cycles.)

We next show that $E_{r}^{s, t}\left(B_{1}(k)\right)$ collapses from $E_{2}$ on (still assuming $t-s \leqslant 2 k$ ). This will be accomplished through a comparison with the Adams spectral sequence for $B(k)$.

Recall from (2.12), that, for the map $i_{k}: B_{1}(k) \rightarrow B(k)$, the induced homomorphism $\left(i_{k}\right)^{*}: H^{*} B(k) \rightarrow H^{*} B_{1}(k)$ is the canonical projection $M(k) \rightarrow M_{1}(k)$. Thus, $\left(i_{k}\right)^{*}$ is covered by a map of resolutions $\mathscr{D} \rightarrow \mathscr{C}$ :

$$
\begin{array}{llccccccccc}
\cdots & \rightarrow & D_{q} & \rightarrow & D_{q-1} & \rightarrow & \cdots & \rightarrow & D_{0} & \rightarrow & M(k) \rightarrow 0 \\
& & \downarrow & & \downarrow & & & & \downarrow & & \downarrow \\
\cdots & \rightarrow & C_{q} & \rightarrow & C_{q-1} & \rightarrow & \cdots & \rightarrow & C_{0} & \rightarrow & M_{1}(k) \rightarrow 0
\end{array}
$$

(By definition, $D_{q} \subset C_{q}$, and the maps $D_{q} \rightarrow C_{q}$ above are the inclusions.) This in turn induces a map of spectral sequences $\left(i_{k}\right)_{*}: E_{r}\left(B_{1}(k)\right) \rightarrow E_{r}(B(k))$. Lemma (4.6) identifies $E_{2}^{s, t}\left(B_{1}(k)\right)$ as a subcomplex of $E_{2}^{s . t}(B(k))$ when $1 \leqslant t-s \leqslant 2 k$; that is, $\left(i_{k}\right)_{*}: E_{2}^{s . t}\left(B_{1}(k)\right) \rightarrow E_{2}^{s . t}(B(k))$ is injective in this range of total dimensions. But, according to (4.2)(a), when $1 \leqslant t-s \leqslant 2 k, E_{2}^{s . t}(B(k))=E_{\infty}^{s . t}(B(k))$, i.e, the spectral sequence collapses. As a subcomplex, $E_{r}^{s . t}\left(B_{1}(k)\right)$ must behave in the same way, and this enables us to write down $E_{\infty}^{s . t}\left(B_{1}(k)\right)$.

Lemma (4.8). $E_{2}^{s, t}\left(B_{1}(k)\right)=E_{\infty}^{s, t}\left(B_{1}(k)\right)$ for $t-s \leqslant 2 k$.

Corollary (4.9). $\left(i_{k}\right)_{*}: \pi_{q}\left(B_{1}(k)\right) \rightarrow \pi_{q}(B(k))$ is injective if $1 \leqslant q \leqslant 2 k$.

Proof. The preceding arguments show that $\left(i_{k}\right)_{*}: E_{\propto}^{s . t}\left(B_{1}(k)\right) \rightarrow E_{\propto}^{s . t}(B(k))$ is injective for $1 \leqslant t-s \leqslant 2 k$. The corollary follows by an easy induction over the Adams filtration.

We now close this discussion by listing some of the homotopy groups of $B_{1}(k)$.

Let $Z_{\hat{2}}=\lim _{\leftarrow} Z_{2^{\prime}}$ denote the 2-adic integers.

THEOREM (4.10). (a) $\pi_{0}\left(B_{1}(k)\right)=Z_{\hat{2}}$.

(b) All elements of $\pi_{q}\left(B_{1}(2 k)\right)=\pi_{q}\left(B_{1}(2 k+1)\right), 1 \leqslant q \leqslant 4 k+2$, are of order 2 . $A Z_{2}$-basis for these groups is in one-to-one correspondence with $\left\{\lambda_{I} \mid I\right.$ admissible, $\left.\operatorname{dim} \lambda_{I} \leqslant 4 k+2, t(I) \geqslant 2 k+1, t(I)=1(\bmod 2)\right\}$.

Proof. Recall that in the $\Lambda$-algebra, left multiplication by $\lambda_{0}$ corresponds to precomposition with the degree 2 map $S^{0} \rightarrow S^{0}$, i.e., $\lambda_{0}=h_{0}$ in the usual Adams 
spectral sequence notation. Thus, a nontrivial left multiplication by $\lambda_{0}$ in $E_{\propto}$ indicates the presence of a nontrivial extension.

Statement (a) of the theorem now follows directly by looking at the tower of elements $\lambda_{0}^{j}, j \geqslant 0$, of total dimension 0 in $E_{\infty}\left(B_{1}(k)\right)$.

To prove (b), we use the cofibration of spectra

$$
B_{1}(2 k)=B_{1}(2 k+1) \stackrel{2}{\rightarrow} B_{1}(2 k+1) \stackrel{i_{2 k+1}}{\rightarrow} B(2 k+1),
$$

the existence of which was verified in (2.15). This yields a long exact sequence of homotopy groups

$$
\begin{aligned}
\cdots \rightarrow \pi_{q} B_{1}(2 k+1) \stackrel{2}{\rightarrow} & \pi_{q} B_{1}(2 k+1) \stackrel{\left(i_{2 k+1}\right)_{*}}{\rightarrow} \pi_{q} B(2 k+1) \\
& \rightarrow \pi_{q-1} B_{1}(2 k+1) \rightarrow \cdots
\end{aligned}
$$

By (4.9), $\left(i_{2 k+1}\right)_{*}: \pi_{q}\left(B_{1}(2 k+1)\right) \rightarrow \pi_{q}(B(2 k+1))$ is injective for $1 \leqslant q \leqslant 4 k+2$. Hence, $2 \pi_{q}\left(B_{1}(2 k+1)\right)=0$ by exactness, i.e, all elements have order 2 .

The statement concerning a $Z_{2}$-basis for these groups is a consequence of the description of $E_{\infty}^{s, t}\left(B_{1}(k)\right)=E_{2}^{s, t}\left(B_{1}(k)\right)$ given in (4.6).

REMARKS. (1) Recall that the group structure of $\pi_{2 k}(B(k))$ has not been completely determined. Property (b) of (4.1) identifies a rather large subgroup, namely, $\left(i_{k}\right)_{*}\left(\pi_{2 k}\left(B_{1}(k)\right)\right)$, as consisting entirely of elements of order 2 .

(2) Outside the range of dimensions handled in (4.10), the groups $\pi_{q}\left(B_{1}(k)\right)$ will have elements of order greater than 2. For instance, let $f \in \pi_{2^{j+1}}\left(B\left(2^{j}\right)\right)$ be a map represented by $\lambda_{2^{\prime+1}}$, as discussed in the remark following (4.2).

Consider the cofibration

$$
B_{1}\left(2^{j}-1\right) \rightarrow B_{1}\left(2^{j}\right) \stackrel{i_{2 \prime}^{\prime}}{\rightarrow} B\left(2^{j}\right) .
$$

Since $\lambda_{0}^{n} \lambda_{2,+1}=\lambda_{2,-n+1}\left(\lambda_{2, n+1} \lambda_{2, n \cdot 2} \cdots \lambda_{2}\right)$ in $E_{\infty}\left(B\left(2^{j}\right)\right)$, the spectral sequence calculations given earlier show that neither $f$ nor any of its multiples can lie in the image of

$$
\left(i_{2^{\prime}}\right)_{*}: \pi_{2^{\prime+1}}\left(B_{1}\left(2^{j}\right)\right) \rightarrow \pi_{2^{j+1}}\left(B\left(2^{j}\right)\right) .
$$

Thus, from the long exact sequence

$$
\cdots \rightarrow \pi_{q}\left(B_{1}\left(2^{j}-1\right)\right) \rightarrow \pi_{q}\left(B_{1}\left(2^{j}\right)\right) \stackrel{\left(i_{2}\right)_{*}}{\rightarrow} \pi_{q}\left(B\left(2^{j}\right)\right) \stackrel{\delta}{\rightarrow} \pi_{q-1}\left(B_{1}\left(2^{j}-1\right)\right) \rightarrow \cdots,
$$

one sees that $\delta f \in \pi_{2^{j+1}-1}\left(B_{1}\left(2^{j}-1\right)\right)$ has order $2^{j+2}$.

5. $B_{1}(k)$ representability of homology classes. Given spectra $E$ and $X, E_{n}(X)$ and $E^{n}(X)$ will denote the $n$th generalized homology and cohomology groups of $X$ with respect to $E$, e.g., $E_{n}(X)=\pi_{n}(E \wedge X)$.

Recall from $\S 2$ the commutative diagram:

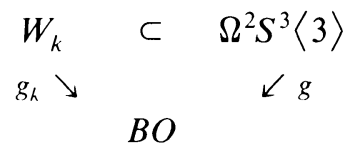


The inclusion $W_{k} \subset \Omega^{2} S^{3}\langle 3\rangle$ induces a map of Thom spectra $T\left(g_{k}\right) \rightarrow T(g)$. Completing this at 2 and using (2.10) then yields a map: $j: B_{1}(k) \rightarrow K\left(Z_{\hat{2}}\right)$ representing the Thom class of $B_{1}(k)$.

The object of this section is to prove

THEOREM (5.1). For any $C W$ complex $X$,

$$
j_{*}: B_{1}(k)_{n}(X) \rightarrow H_{n}\left(X ; Z_{\hat{2}}\right)
$$

is surjective, provided that $n<2 k+2$.

The proof will be given in $\$ 5.2$. The arguments in this proof can then be easily used to discover conditions which characterize $B_{1}(k)$, and this process is carried out in $§ 5.3$.

An immediate consequence of (5.1) is

Corollary (5.2). Suppose that $M$ is a Z-orientable, closed $n$-manifold. Let $\nu$ be its stable normal bundle, denote the Thom spectrum by $T(\nu)$, and let $U_{Z}: T(\nu) \rightarrow K\left(Z_{\hat{2}}\right)$ represent the Thom class. Then $M$ is $B_{1}([n / 2])$-orientable in the sense that there is a class $U_{B} \in B_{1}([n / 2])^{0}(T(\nu))=\left[T(\nu), B_{1}([n / 2])\right]$ such that $j_{*} U_{B}=U_{Z}$, i.e., such that the diagram

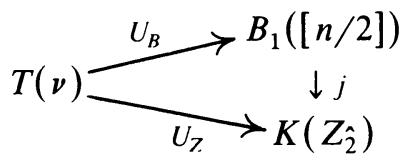

commutes.

Proof. It is well known that the suspension spectrum of $M^{+}$is $S$-dual to $T(\nu)$. $\left(M^{+}\right.$means $M$ plus a disjoint base point.) Now, by (5.1), $j_{*}: B_{1}[n / 2]_{n}\left(M^{+}\right) \rightarrow$ $H_{n}\left(M^{+} ; Z_{\hat{2}}\right)$ is surjective so that, under $S$-duality, $j_{*}: B_{1}[n / 2]^{0}(T(\nu)) \rightarrow$ $H^{0}\left(T(\nu) ; Z_{\hat{2}}\right)$ must be surjective, too. The result follows by pulling back the Thom class $U_{\mathrm{Z}}$.

REMARK. Let $U_{\nu_{M}} \in H^{0}(T(\nu))$ denote the mod 2 Thom class, and let $I_{n}=\{a \in$ $A \mid a U_{\nu_{M}}=0$ for all $Z$-orientable, closed $n$-manifolds $\left.M\right\}$. For instance, $\mathrm{Sq}^{1} \in I_{n}$ for any $n$. A fairly straightforward calculation, utilizing the connection between $S$-duality and the canonical antiautomorphism $\chi: A \rightarrow A$, shows that

$$
A\left\{\mathrm{Sq}^{1}, \chi\left(\mathrm{Sq}^{i}\right) \mid i>[n / 2]\right\} \subset I_{n} \text {. }
$$

Thus, for any $Z$-orientable, closed $n$-manifold, there is always an algebraic factorization of $U_{Z}^{*}$

$$
\begin{aligned}
& M_{1}[n / 2] \\
& j^{*} \nearrow \quad \searrow 0 \\
& A / A\left\{\mathrm{Sq}^{1}\right\} \stackrel{U_{2}^{*}}{\rightarrow} \quad H^{*}(T(\nu))
\end{aligned}
$$


where $j^{*}$ is the projection. (5.2) then says that one can geometrically realize the homomorphism $v$ above by a $B_{1}[n / 2]$ Thom class $U_{B}$.

Furthermore, $I_{n}$ has been determined explicitly by Brown and Peterson [5], and, when $n \neq 0(\bmod 4)$, their calculations show that $I_{n}=A\left\{\mathrm{Sq}^{1}, \chi\left(\mathrm{Sq}^{i}\right) \mid i>[n / 2]\right\}$. Thus, for such $n, B_{1}[n / 2]$ has the smallest possible cohomology for any spectrum possessing the orientability property of (5.2), meaning that, in this sense, (5.2) is best possible.

As usual, the results here are analogous to properties of the Brown-Gitler spectrum $B(k)$. This time, the corresponding theorems, due to Brown and Gitler, are

THeOREM (5.3) [3]. If $\alpha: B(k) \rightarrow K\left(Z_{2}\right)$ is the map representing $1 \in H^{*} B(k)=$ $M(k)$, then, for any $C W$ complex $X$,

$$
\alpha_{*}: B(k)_{n}(X) \rightarrow H_{n} X
$$

is surjective, provided that $n<2 k+2$.

COROLlary (5.4) [3]. Suppose that $M$ is any closed n-manifold and $U: T(\nu) \rightarrow K\left(Z_{2}\right)$ represents the $(\bmod 2)$ Thom class. Then $M$ is $B[n / 2]$-orientable in the sense that there is a class $\tilde{U} \in B[n / 2]^{0}(T(\nu))$ such that $\alpha_{*} \tilde{U}=U$.

5.1. Background and notation. In this subsection, we organize the material needed to prove (5.1).

First of all. Brown and Gitler actually proved a result which is slightly stronger than (5.3), and this stronger version is what we shall use in the proof of (5.1). To state it, we need to review a few details about the way that $B(k)$ is constructed.

Let $\mathscr{D}=\mathscr{D}(k)$ denote the $A$-free resolution of $H^{*} B(k)$ described in (3.5):

$$
\text { D: } \cdots \rightarrow D_{q} \stackrel{d}{\rightarrow} D_{q-1} \rightarrow \cdots \rightarrow D_{0} \stackrel{\varepsilon}{\rightarrow} M(k) \rightarrow 0 .
$$

Based on this resolution, Brown and Gitler explicitly constructed a generalized Postnikov tower $\mathscr{D}_{T}$ for $B(k)$ :

$$
\begin{aligned}
& \mathscr{D}_{T} \text { : } \\
& Y_{q} \stackrel{h_{q+1}}{\rightarrow} L_{q+1} \\
& \alpha_{4} \downarrow \\
& Y_{q-1} \stackrel{h_{q}}{\rightarrow} L_{q} \\
& \downarrow \\
& \downarrow \\
& Y_{0}=L_{0} \stackrel{h_{1}}{\rightarrow} L_{1}
\end{aligned}
$$


That is, the $Y_{q}$ are $\Omega$-spectra, and the $L_{q}$ are generalized Eilenberg-Mac Lane spectra such that $\pi_{*} L_{q}$ is a graded vector space over $Z_{2}$ and $H^{*} L_{q}=D_{q}$. Also, $\alpha_{q}$ : $Y_{q} \rightarrow Y_{q-1}$ is the fibration with fibre $L_{q}$ induced by $h_{q}$ from the contractible fibration over $L_{q}$. $\left(h_{q}\right.$ is a morphism of degree one, i.e., $\left(h_{q}\right)_{t}:\left(Y_{q-1}\right)_{t} \rightarrow:\left(L_{q}\right)_{t+1}$. If $\varepsilon_{q}: L_{q} \rightarrow Y_{q}$ denotes the inclusion of the fibre, then $\left(h_{q+1} \varepsilon_{q}\right)^{*}: H^{*} L_{q+1} \rightarrow H^{*} L_{q}$ is the same as the differential $d: D_{q+1} \rightarrow D_{q}$. Note that the base spectrum of the tower, $L_{0}$, satisfies $H^{*} L_{0}=D_{0}=A$, and hence $L_{0}=K\left(Z_{2}\right)$.

With this machinery in place, $B(k)$ is defined as the limit space

$$
B(k)=Y_{\infty}=\lim _{\leftarrow} Y_{q} .
$$

The construction of the tower $\mathscr{D}_{T}$ is carefully controlled to possess the following property.

Lemma (5.5). For any $C W$ complex $X$,

$$
\left(\alpha_{q}\right)_{*}:\left(Y_{q}\right)_{n}(X) \rightarrow\left(Y_{q-1}\right)_{n}(X)
$$

is surjective, provided that $n<2 k+2$.

Proof. Given an abelian group $G$, define $\operatorname{ch}(G)=\operatorname{Hom}(G, R / Z)$. In [3], Brown and Gitler discuss a functor $\chi$ on spectra, the Pontrjagin duality functor, whose main property is that, for any spectrum $h$ and $\mathrm{CW}$ complex $X$,

$$
\chi(h)^{n}(X)=\operatorname{ch}\left(h_{n}(X)\right)
$$

(assuming that $h_{n}\left(S^{0}\right)$ is finite for all $n$ ).

Applying $\chi$ to the fibration $L_{q} \rightarrow Y_{q} \stackrel{\alpha_{q}}{\rightarrow} Y_{q-1}$ then produces another fibration $\chi\left(Y_{q-1}\right) \stackrel{\chi\left(\alpha_{q}\right)}{\rightarrow} \chi\left(Y_{q}\right) \rightarrow \chi\left(L_{q}\right)$ induced by $\chi\left(h_{q}\right): \chi\left(L_{q}\right) \rightarrow \chi\left(Y_{q-1}\right)$. An important detail in Brown and Gitler's work is that $\chi\left(h_{q}\right)_{n}: \chi\left(L_{q}\right)_{n} \rightarrow \chi\left(Y_{q-1}\right)_{n+1}$ is zero when $n<2 k+1$ (see [3, Theorem 5.1(iv)]). Therefore, $\chi\left(\alpha_{q}\right)_{*}: \chi\left(Y_{q-1}\right)^{n}(X) \rightarrow$ $\chi\left(Y_{q}\right)^{n}(X)$ is injective for $n<2 k+2$. Equivalently, after applying (5.6), $\left(\alpha_{q}\right)_{*}$ : $\left(Y_{q}\right)_{n}(X) \rightarrow\left(Y_{q-1}\right)_{n}(X)$ is surjective for $n<2 k+2$.

(5.5) can also be stated more directly in terms of Postnikov towers. Namely, consider the complex

$$
\begin{aligned}
\mathscr{D} \otimes H^{*} X: \cdots & \rightarrow D_{q} \otimes H^{*} X \stackrel{d \otimes \text { id }}{\rightarrow} D_{q-1} \otimes H^{*} X \rightarrow \cdots \\
& \rightarrow D_{0} \otimes H^{*} X \stackrel{\varepsilon \otimes \text { id }}{\rightarrow} M(k) \otimes H^{*} X \rightarrow 0 .
\end{aligned}
$$

This is an $A$-free resolution of $H^{*}(B(k) \wedge X)=M(k) \otimes H^{*} X$. (Here, each $D_{q} \otimes$ $H^{*} X$ has the diagonal $A$-module structure, that is, $a(x \otimes y)=\sum a_{i}^{\prime} x \otimes a_{i}^{\prime \prime} y$, and an argument involving the formula $\chi(a) \lambda^{I} \otimes y=\sum \chi\left(a_{i}^{\prime}\right)\left(\lambda^{I} \otimes a_{i}^{\prime \prime} y\right)$ shows that these 
modules are in fact free over $A$.) Associated to this resolution is a Postnikov tower $\mathscr{D}_{T} \wedge X$ with limit space $B(k) \wedge X$ :

$$
B(k) \wedge X
$$

$$
\mathscr{D}_{T} \wedge X:
$$

$$
\begin{aligned}
& Y_{q} \wedge X \stackrel{h_{q+1} \wedge \text { id }}{\rightarrow} \quad L_{q+1} \wedge X \\
& \alpha_{4} \wedge \text { id } \downarrow \\
& Y_{q-1} \wedge X \quad \stackrel{h_{4} \wedge \text { id }}{\rightarrow} \quad L_{q} \wedge X \\
& Y_{0} \wedge X \quad \stackrel{h_{1} \wedge \text { id }}{\rightarrow} \quad L_{1} \wedge X
\end{aligned}
$$

The content of (5.5) is that any map $u$ : $S^{n} \rightarrow Y_{q-1} \wedge X, n<2 k+2$, lifts up the tower to $Y_{q} \wedge X$ (and hence, by induction, all the way up to $B(k) \wedge X$ ). Of course, if one happens to start with $u: S^{n} \rightarrow Y_{0} \wedge X=K\left(Z_{2}\right) \wedge X$, the result is (5.3).

The proof of (5.1) will make use of the tower $\mathscr{D}_{T} \wedge X$, as well as similar towers for $B_{1}(k) \wedge X$ and $K\left(Z_{\hat{2}}\right) \wedge X$.

The following notation of modules and spectra shall be preserved for the rest of the section.

First, let $\mathscr{C}=\mathscr{C}(k)$ denote the free resolution for $M_{1}(k)$ described in (3.10):

$$
\mathscr{C}: \cdots \rightarrow C_{q} \stackrel{d}{\rightarrow} C_{q-1} \rightarrow \cdots \rightarrow C_{0} \stackrel{\varepsilon}{\rightarrow} M_{1}(k) \rightarrow 0 ;
$$

and let $\mathscr{C}_{T}$ denote a Postnikov tower associated to it with limit space $B_{1}(k)$ (recall that $B_{1}(k)$ was defined as a 2-completion):

$$
\begin{array}{cccc} 
& B_{1}(k) & & \\
\mathscr{C}_{T}: & \vdots & & \\
& X_{q} & \stackrel{k_{q+1}}{\rightarrow} & K_{q+1} \\
\downarrow & & \\
X_{q-1} & \stackrel{k_{q}}{\rightarrow} & K_{q} \\
\downarrow & & \\
\vdots & & \\
\downarrow & & \\
X_{0} & =K_{0} & \stackrel{k_{1}}{\rightarrow} & K_{1}
\end{array}
$$

As with $\mathscr{D}_{T}$, at the bottom of the tower, one has $X_{0}=K\left(Z_{2}\right)$. 
Similarly, let $\mathscr{E}=\mathscr{E}(k)$ denote the " $k$-acceptable" resolution of $H^{*}\left(K\left(Z_{\hat{2}}\right)\right)=$ $A / A\left\{\mathrm{Sq}^{1}\right\}$ given by (3.15):

$$
\mathscr{E}: \cdots \rightarrow E_{q} \stackrel{d}{\rightarrow} E_{q-1} \rightarrow \cdots \rightarrow E_{0} \stackrel{\varepsilon}{\rightarrow} A / A\left\{\mathrm{Sq}^{1}\right\} \rightarrow 0 ;
$$

and let $\mathscr{E}_{T}$ denote a Postnikov tower based on this resolution having as limit space the 2-complete spectrum $K\left(Z_{\hat{2}}\right)$ :

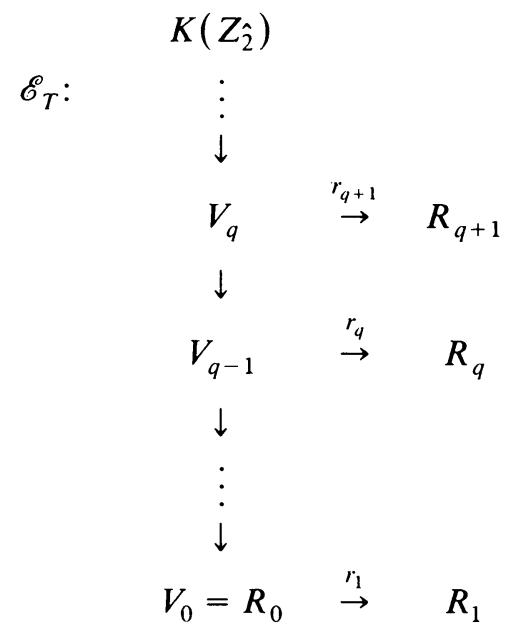

In particular, $V_{0}=K\left(Z_{2}\right)$.

In the previous resolution, one can tensor all modules with $H^{*} X$ and differentials with the identity in order to obtain new resolutions, denoted by $\mathscr{C} \otimes H^{*} X$ and $\mathscr{E} \otimes H^{*} X$. These in turn give rise to new Postnikov towers, denoted $\mathscr{C}_{T} \wedge X$ and $\mathscr{E}_{T} \wedge X$, obtained by smashing all spectra with $X$ and all maps with the identity.

Now, recall from (2.12) that the map $i_{k}: B_{1}(k) \rightarrow B(k)$ produces a homomorphism in cohomology, $\left(i_{k}\right)^{*}: H^{*} B(k) \rightarrow H^{*} B_{1}(k)$, which is the obvious projection $M(k) \rightarrow M_{1}(k)$. Thus, $\left(i_{k}\right)^{*} \otimes$ id is covered by a map of resolutions $\mathscr{D} \otimes H^{*} X \rightarrow \mathscr{C}$ $\otimes H^{*} X$ :

$$
\begin{aligned}
& \cdots \rightarrow D_{q} \otimes H^{*} X \rightarrow D_{q-1} \otimes H^{*} X \rightarrow \cdots \rightarrow D_{0} \otimes H^{*} X \rightarrow M(k) \otimes H^{*} X \rightarrow 0
\end{aligned}
$$

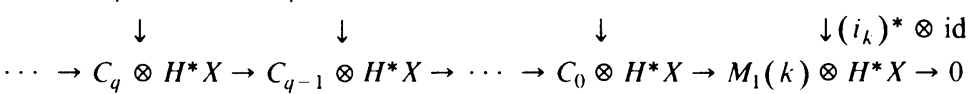

(Recall that, by definition, $D_{q} \subset C_{q}$, and the vertical maps are the inclusions.) Then, by naturality, there is an associated map of Postnikov towers $\left(i_{k}\right)_{T} \wedge$ id: $\mathscr{C}_{T} \wedge X \rightarrow$ $\mathscr{D}_{T} \wedge X$ : 


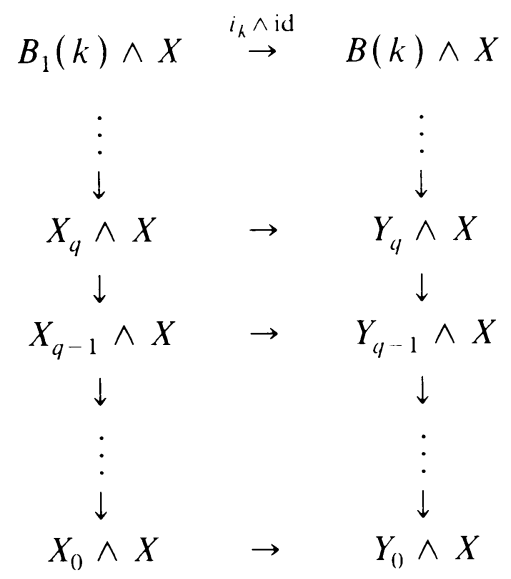

Similarly, for the map $j: B_{1}(k) \rightarrow K\left(Z_{\hat{2}}\right)$, the induced homomorphism $j^{*}$ : $H^{*}\left(K\left(Z_{\hat{2}}\right)\right) \rightarrow H^{*} B_{1}(k)$ is the projection $A / A\left\{\mathrm{Sq}^{1}\right\} \rightarrow M_{1}(k)$. Thus, there is an obvious map of resolutions $\mathscr{E} \otimes H^{*} X \rightarrow \mathscr{C} \otimes H^{*} X$ covering $j^{*} \otimes$ id. (By definition, $E_{q} \subset C_{q}$, so that $\mathscr{E}$ maps to $\mathscr{C}$ by inclusion.) And, once again, one winds up with an associated map of Postnikov towers $j_{T} \wedge$ id: $\mathscr{C}_{T} \wedge X \rightarrow \mathscr{E}_{T} \wedge X$.

The idea is that all this notation can be molded into a sensible proof of (5.1).

5.2. Proof of Theorem (5.1). To begin the proof of (5.1), let $w \in H_{n}\left(X ; Z_{\hat{2}}\right)$ be given, $n<2 k+2$. Represent $w$ as a map $w: S^{n} \rightarrow K\left(Z_{\hat{2}}\right) \wedge X$. Then $w$ can be regarded as a coherent sequence of maps $w_{q}: S^{n} \rightarrow V_{q} \wedge X$ up the tower $\mathscr{E}_{T} \wedge X$ :

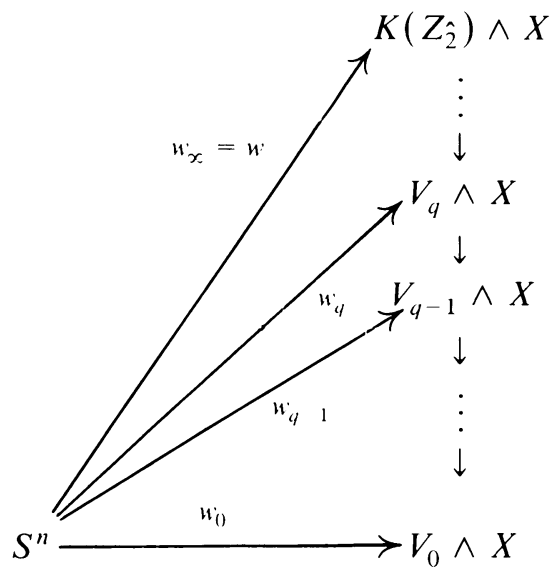

Note that $w_{0}: S^{n} \rightarrow V_{0} \wedge X=K\left(Z_{2}\right) \wedge X$ represents the mod 2 reduction of the homology class $w$.

We want to find $u \in B_{1}(k)_{n}(X)$ (i.e., $u: S^{n} \rightarrow B_{1}(k) \wedge X$ ) such that $j_{*} u=w$. The strategy will be to show inductively that $w_{0}$ lifts up the tower $\mathscr{C}_{T} \wedge X$ all the way to $B_{1}(k) \wedge X$ in a manner compatible with the class $w$. That is, we shall complete the diagram: 


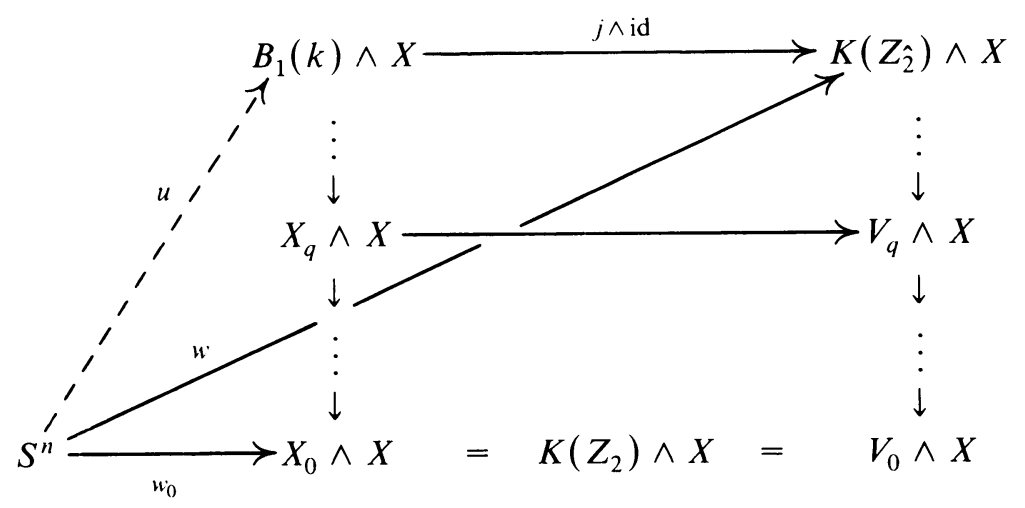

Inductive assumption. Assume that there exist maps $u_{0}, u_{1}, \ldots, u_{q}$, where $u_{i}$ : $S^{n} \rightarrow X_{i} \wedge X$, which make the following diagrams $\left(P_{q}\right)$ and $\left(Q_{q}\right)$ commute:

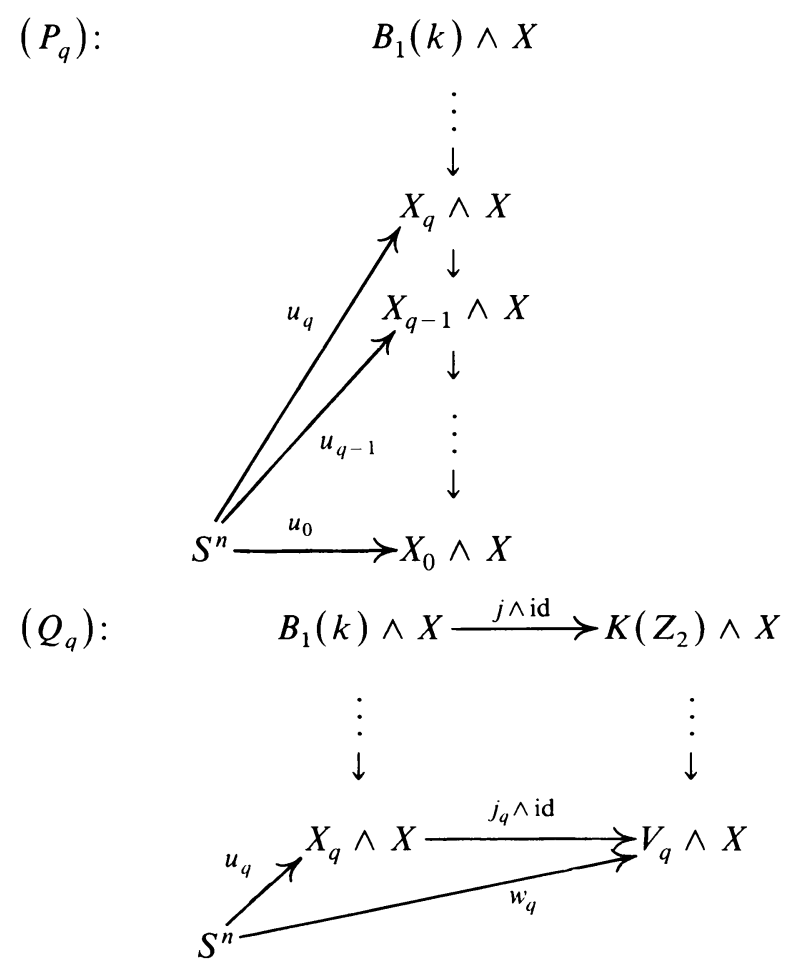

Begin the induction by taking $u_{0}=w_{0}$.

Next, assume that $u_{0}, \ldots, u_{q-1}$ have been found satisfying $\left(P_{q-1}\right)$ and $\left(Q_{q-1}\right)$. We shall describe how to choose $u_{q}$ so that $\left(P_{q}\right)$ and $\left(Q_{q}\right)$ hold.

$\left(P_{q}\right)$ Showing that $u_{q-1}$ lifts to $X_{q} \wedge X$ is equivalent to verifying that $k_{q}^{\prime} u_{q-1}=0$, where $k_{q}^{\prime}=k_{q} \wedge$ id is the $k$-invariant for $X_{q} \wedge X \rightarrow X_{q-1} \wedge X$ in the tower $\mathscr{C}_{T} \wedge X$ : 


$$
\begin{aligned}
B_{1}(k) & \wedge X \\
\vdots & \\
\downarrow & \\
X_{q} & \wedge X \\
& \\
S^{n} \stackrel{\downarrow}{\rightarrow} \quad X_{q-1} & \wedge X \stackrel{k_{q}^{\prime}}{\rightarrow} K_{q} \wedge X
\end{aligned}
$$

Since $K_{q} \wedge X$ is a generalized $Z_{2}$ Eilenberg-Mac Lane spectrum, this is the same as showing that $\left(k_{q}^{\prime} u_{q-1}\right)^{*}=0$ in cohomology, i.e., that $\left(k_{q}^{\prime} u_{q-1}\right)^{*}\left(\lambda^{I} \otimes x\right)=0$ for all $A$-basis elements $\lambda^{I} \in H^{*} K_{q}=C_{q}$ and all $x \in H^{*} X$.

Recall that $C_{q}$ has two types of basis elements, namely, $\lambda^{I}$ with $l(I)=q$ such that either $t(I) \geqslant k$ or $I$ is $k$-acceptable.

First, suppose that $t(I) \geqslant k$. Then, as part of the mapping of towers $\left(i_{k}\right)_{T} \wedge$ id: $\mathscr{C}_{T} \wedge X \rightarrow \mathscr{D}_{T} \wedge X$, there is a commutative diagram involving the $k$-invariants $k_{q}^{\prime}$ and $h_{q}^{\prime}=h_{q} \wedge$ id:

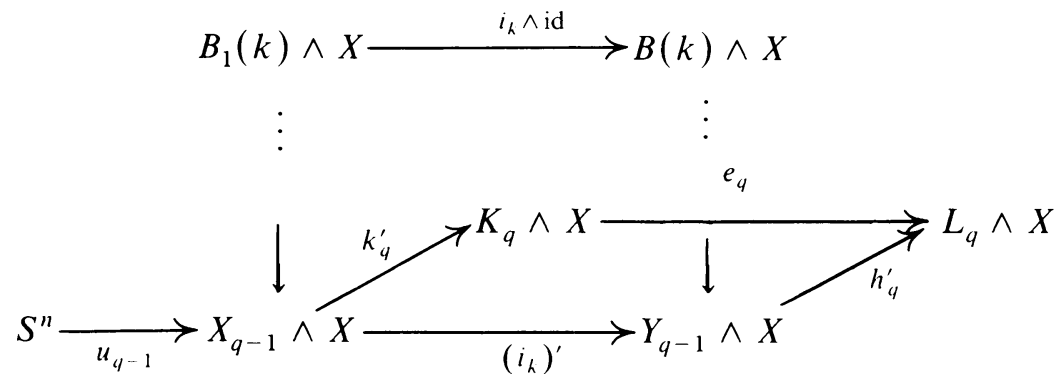

where $\left(e_{q}\right)^{*}: H^{*}\left(L_{q} \wedge X\right) \rightarrow H^{*}\left(K_{q} \wedge X\right)$ is the inclusion $D_{q} \otimes H^{*} X \rightarrow C_{q} \otimes H^{*} X$. Now, by (5.5), $\left(i_{k}\right)^{\prime} u_{q-1}$ lifts to $Y_{q} \wedge X$. Hence, $h_{q}^{\prime}\left(i_{k}\right)^{\prime} u_{q-1}=0$, so that

$$
0=\left(e_{q} k_{q}^{\prime} u_{q-1}\right) *\left(\lambda^{I} \otimes x\right)=\left(k_{q}^{\prime} u_{q-1}\right) * e_{q}^{*}\left(\lambda^{I} \otimes x\right)=\left(k_{q}^{\prime} u_{q-1}\right) *\left(\lambda^{I} \otimes x\right)
$$

as desired.

Secondly, suppose that $I$ is $k$-acceptable. Then, as part of the mapping of towers $j_{T} \wedge$ id: $\mathscr{C}_{T} \wedge X \rightarrow \mathscr{E}_{T} \wedge X$, there is a commutative diagram involving the $k$-invariants $k_{q}^{\prime}$ and $r_{q}^{\prime}=r_{q} \wedge$ id:

$$
B_{1}(k) \wedge X \longrightarrow K\left(Z_{\hat{2}}\right) \wedge X
$$

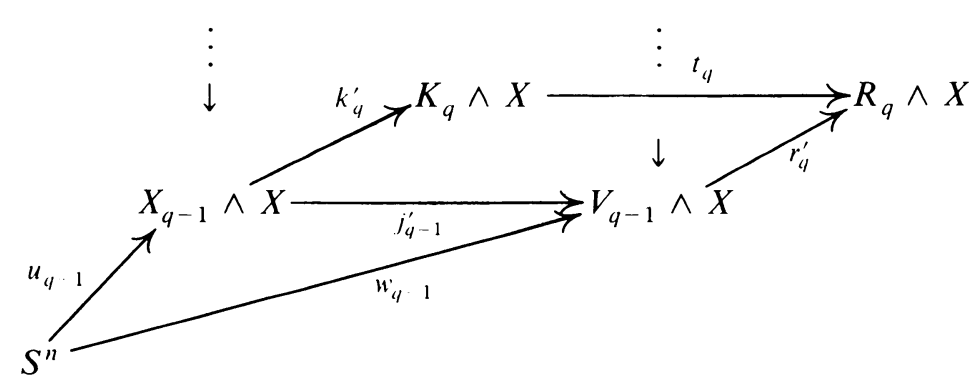


where $\left(t_{q}\right)^{*}: H^{*}\left(R_{q} \wedge X\right) \rightarrow H^{*}\left(K_{q} \wedge X\right)$ is the inclusion $E_{q} \otimes H^{*} X \rightarrow C_{q} \otimes H^{*} X$ and $j_{q-1}^{\prime}=j_{q-1} \wedge$ id. (Note that $w_{q-1}=j_{q-1}^{\prime} u_{q-1}$ by induction.) Since $w_{q-1}$ lifts to $V_{q} \wedge X$ as $w_{q}$, it follows that $r_{q}^{\prime} j_{q-1}^{\prime} u_{q-1}=r_{q}^{\prime} w_{q-1}=0$. Therefore,

$$
0=\left(t_{q} k_{q}^{\prime} u_{q-1}\right) *\left(\lambda^{I} \otimes x\right)=\left(k_{q}^{\prime} u_{q-1}\right)^{*} t_{q}^{*}\left(\lambda^{I} \otimes x\right)=\left(k_{q}^{\prime} u_{q-1}\right) *\left(\lambda^{I} \otimes x\right) .
$$

These calculations show that $k_{q}^{\prime} u_{q-1}=0$. Consequently, there exists $z_{q}: S^{n} \rightarrow$ $X_{q} \wedge X$ satisfying property $\left(P_{q}\right)$.

$\left(Q_{q}\right)$ Consider the commutative diagram involving $j_{T} \wedge$ id: $\mathscr{C}_{T} \wedge X \rightarrow \mathscr{E}_{T} \wedge X$ :

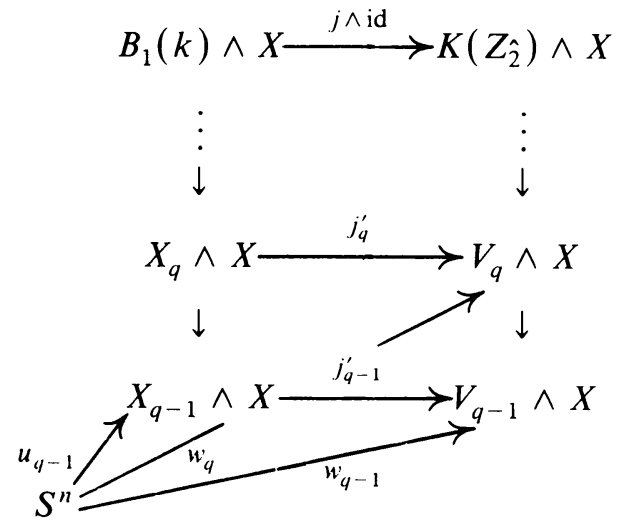

Recall that $K_{q} \wedge X$ denotes the fibre of $X_{q} \wedge X \rightarrow X_{q-1} \wedge X$ and $R_{q} \wedge X$ denotes the fibre of $V_{q} \wedge X \rightarrow V_{q-1} \wedge X$.

We would like to check if $j_{q}^{\prime} z_{q}$ and $w_{q}$ are equal. If not, then as two liftings of $w_{q-1}$, they must differ by a map $\varepsilon$ into the fibre $R_{q} \wedge X$, i.e., $w_{q}=j_{q}^{\prime} z_{q}+\varepsilon$ for some $\varepsilon$ : $S^{n} \rightarrow R_{q} \wedge X$. But since $H^{*}\left(R_{q} \wedge X\right)=E_{q} \otimes H^{*} X \subset C_{q} \otimes H^{*} X=H^{*}\left(K_{q} \wedge X\right)$, such an $\varepsilon$ would factor as $S^{n} \stackrel{\varepsilon^{\prime}}{\rightarrow} K_{q} \wedge X \stackrel{t_{q}}{\rightarrow} R_{q} \wedge X$. Let $\varepsilon^{\prime \prime}$ denote the composition of $\varepsilon^{\prime}$ with the inclusion of the fiber $K_{q} \wedge X \rightarrow X_{q} \wedge X$. Then by letting $u_{q}=z_{q}+\varepsilon^{\prime \prime}$, one obtains a map satisfying property $\left(Q_{q}\right)$.

We should remark that this map, $u_{q}$, will still satisfy property $\left(P_{q}\right)$, since $z_{q}$ and $u_{q}$ differ by a map into $K_{q} \wedge X$ and the composition $K_{q} \wedge X \rightarrow X_{q} \wedge X \rightarrow X_{q-1} \wedge X$ is zero.

This completes the induction.

Set $u=u_{\infty}$, and it is clear that $j_{*} u=w$.

5.3. Homotopy characterizations of $B_{1}(k)$. Our goal in this subsection is to find necessary and sufficient conditions which determine whether a given spectrum is homotopy 2-equivalent to the spectrum $B_{1}(k)$. In [6], Brown and Peterson formulated various sets of conditions of this type for the Brown-Gitler spectrum $B(k)$. For example, they proved that a spectrum $Y$ is homotopy 2-equivalent to $B(k)$ if and only if $H^{*} Y=M(k)$ and the homology surjectivity of (5.3) remains valid with $Y$ in place of $B(k)$. The proof of this result, as well as other results like it, depended on properties of the Postnikov tower $\mathscr{D}_{T}$ together with a rather exotic condition on manifolds related to Brown and Peterson's earlier work on characteristic classes [5]. 
Our approach here will be to apply Brown and Peterson's methods to the tower $\mathscr{C}_{T}$ - the arguments involved are quite similar to those used in the proof of (5.1). In the end, this will lead us to the desired characterizations of $B_{1}(k)$.

We begin with a definition motivated by the work of Brown and Peterson [6, p. 289].

Definition (5.7). Suppose that $N$ is a closed $n$-manifold, $\nu$ is its stable normal bundle, $T(\nu)$ is the Thom spectrum of $\nu$, and $v \in H^{p}(T(\nu))$. Then $(N, v)$ is said to be adapted to $M_{1}(k)$ if $n-p<2 k+2$ and

$$
0 \rightarrow A\left\{\mathrm{Sq}^{1}, \chi\left(\mathrm{Sq}^{i}\right) \mid i>k\right\} \rightarrow A \stackrel{r^{* *}}{\rightarrow} H^{*}(T(\nu))
$$

is exact, where $v^{*}(a)=a v$.

The notion of adapted manifolds is somewhat unmotivated at this point. However, we can at least show that it is not vacuous.

LeMma (5.8). There exists a Z-orientable, closed $(2 k+1)$-manifold $Q_{k}$ such that, if $U_{v_{Q_{k}}} \in H^{0}\left(T\left(v_{Q_{k}}\right)\right)$ is the Thom class, then $\left(Q_{k}, U_{v_{Q_{k}}}\right)$ is adapted to $M_{1}(k)$.

Proof. Let $I_{n}=\left\{a \in A \mid a U_{\nu_{M}}=0\right.$ for all Z-orientable, closed $n$-manifolds $\left.M\right\}$. Brown and Peterson [5] showed that

$$
I_{2 k+1}=A\left\{\mathrm{Sq}^{1}, \chi\left(\mathrm{Sq}^{i}\right) \mid i>k\right\} .
$$

Let $\left\{v_{\alpha}\right\}$ denote a $Z_{2}$-basis for $A / I_{2 k+1}=M_{1}(k)$. For each $v_{\alpha}$, choose a $Z$-orientable, closed $(2 k+1)$-manifold $N_{\alpha}$ such that $v_{\alpha} U_{v_{N_{\alpha}}} \neq 0$. Then let $Q_{k}=\#_{\alpha} N_{\alpha}$.

Now, for some notation:

Let $\rho_{q}: V_{x}=K\left(Z_{\hat{2}}\right) \rightarrow V_{q}$ denote the projection down the tower $\mathscr{E}_{T}$. For instance, $\rho_{0}: K\left(Z_{\hat{2}}\right) \rightarrow V_{0}=K\left(Z_{2}\right)$ represents reduction $\bmod 2$.

Also, let $j_{q}: X_{q} \rightarrow V_{q}$ denote the $q$ th stage of the mapping of towers $j_{T}: \mathscr{C}_{T} \rightarrow \mathscr{E}_{T}$.

Definition (5.9). Suppose that $X$ is a spectrum and let $z: X \rightarrow K\left(Z_{\hat{2}}\right)$ be given. Then a map $v: X \rightarrow X_{q}$ is called a $k$-acceptable $q$-lifting of $\rho_{0} z$ if the following diagram commutes:

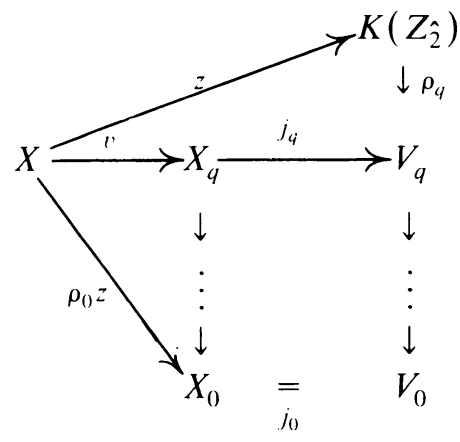

Recall that, in the Postnikov tower $\mathscr{C}_{T}$ for $B_{1}(k)$, if $k_{q}: X_{q-1} \rightarrow K_{q}$ denotes the $k$-invariant and $i: K_{q-1} \rightarrow X_{q-1}$ is the inclusion of the fibre, then $\left(k_{q} i\right)^{*}: H^{*} K_{q} \rightarrow$ $H^{*} K_{q-1}$ realizes the differential $d: C_{q} \rightarrow C_{q-1}$ of the resolution $\mathscr{C}(k)$. We next show 
that this fact, along with the existence of adapted manifolds, actually characterizes the $k$-invariant $k_{q}$.

THEOREM (5.10). Suppose that $N$ is a closed $n$-manifold, $z \in H^{p}\left(T\left(\nu_{N}\right) ; Z_{\hat{2}}\right)$, and $n-p<2 k+2$. Let $v_{q-1}: T\left(\nu_{N}\right) \rightarrow X_{q-1}$ be a k-acceptable $(q-1)$-lifting of $\rho_{0} z$. Then:

(a) $v_{q-1}$ lifts to a $k$-acceptable q-lifting $v_{q}: X \rightarrow X_{q}$ of $\rho_{0} z$.

(b) Furthermore, if $\left(N, \rho_{0} z\right)$ is adapted to $M_{1}(k)$, then $k_{q}$ is the unique map such that $\left(k_{q} i\right)^{*}=d$ and $k_{q} v_{q-1}=0$.

PROOF. The proof of (a) becomes identical to the proof of (5.1) after applying $S$-duality. Hence, we shall only sketch the necessary steps.

First, we may regard $v_{q-1}$ as an element of $\left(X_{q-1}\right)^{p}\left(T\left(\nu_{N}\right)\right)$. Let $u_{q-1}: S^{n-p} \rightarrow$ $X_{q-1} \wedge N^{+}$represent a class in $\left(X_{q-1}\right)_{n-p}\left(N^{+}\right) S$-dual to $v_{q-1}$. Similarly, let $w \in H_{n-p}\left(N^{+} ; Z_{\hat{2}}\right)$ be $S$-dual to $z$. Now, the proof of (5.1) shows that $u_{q-1}$ lifts to a map $u_{q}: S^{n-p} \rightarrow X_{q} \wedge N^{+}$in a way compatible with both the homology class $w$ and the mapping of towers $j_{T} \wedge \mathrm{id}: \mathscr{C}_{T} \wedge N^{+} \rightarrow \mathscr{E}_{T} \wedge N^{+}$. Let $v_{q}: T\left(\nu_{N}\right) \rightarrow X_{q}$ represent a cohomology class in $\left(X_{q}\right)^{p}\left(T\left(\nu_{N}\right)\right) S$-dual to $u_{q}$. Then $v_{q}$ satisfies the conclusion of (a).

To prove (b), note first that $k_{q} v_{q-1}=0$ by (a). Since the tower

$$
\mathscr{C}_{T}: \cdots \rightarrow X_{q} \rightarrow X_{q-1} \rightarrow X_{q-2} \rightarrow \cdots
$$

is constructed from an acyclic resolution of $M_{1}(k)$, the image of $H^{*} X_{q-2}$ in $H^{*} X_{q-1}$ is $M_{1}(k)$; in fact, $H^{*} X_{q-1}=M_{1}(k) \oplus \operatorname{im} k_{q}^{*}$. Thus, the sequence

$$
0 \rightarrow M_{1}(k) \rightarrow H^{*} X_{q-1} \stackrel{i^{*}}{\rightarrow} H^{*} K_{q-1}
$$

is exact. Since $k_{q} v_{q-1}=0, v_{q-1}^{*}: H^{*} X_{q-1} \rightarrow H^{*}\left(T\left(\nu_{N}\right)\right)$ factors through $M_{1}(k)$, splitting the above exact sequence, i.e., there is a commutative diagram of the form:

$$
0 \rightarrow M_{1}(k) \rightarrow H^{*} X_{q-1} \stackrel{i^{*}}{\rightarrow} H^{*} K_{q-1}
$$

(Note that the homomorphism labelled $\tau$ above must be injective because $\left(N, \rho_{0} z\right)$ is adapted to $M_{1}(k)$.) Consequently, $\left(k_{q}\right)^{*}: H^{*} K_{q} \rightarrow H^{*} X_{q-1}$, and hence $k_{q}$, is uniquely determined by the conditions that $k_{q} v_{q-1}=0$ and $\left(k_{q} i\right)^{*}=d$. This proves (b).

Finally, we will use (5.10) to give two characterizations of $B_{1}(k)$.

Corollary (5.11). Suppose that $Y$ is a spectrum such that $H^{*} Y=M_{1}(k)$. In addition, suppose there is a map $z: Y \rightarrow K\left(Z_{\hat{2}}\right)$ such that $\rho_{0} z: Y \rightarrow K\left(Z_{2}\right)$ represents $1 \in H^{*} Y$. If, for some $(N, v)$ adapted to $M_{1}(k)$, there is a map $\tilde{v}: T\left(\nu_{N}\right) \rightarrow Y$ for which $\rho_{0} z \tilde{v}=v$, then $Y$ is homotopy 2-equivalent to $B_{1}(k)$. 
Proof. We will construct $k$-acceptable $q$-liftings of $\rho_{0} z$ by induction on $q$. Assume by induction that $f: Y \rightarrow X_{q-1}$ is a $k$-acceptable $(q-1)$-lifting of $\rho_{0} z$, yielding the following commutative diagram:

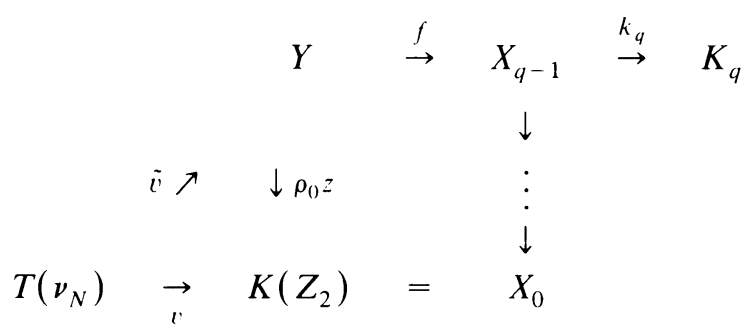

Since $f \tilde{v}$ is a $k$-acceptable $(q-1)$-lifting of $v=\rho_{0}(z \tilde{v}),(5.10)$ (a) implies that $k_{q} f \tilde{v}=0$. Moreover, since $(N, v)$ is adapted to $M_{1}(k), \tilde{v}^{*}: H^{*} Y \rightarrow H^{*}\left(T\left(\nu_{N}\right)\right)$ is injective. Hence, $k_{q} f=0$, and, therefore, $f$ lifts to $\tilde{f}: Y \rightarrow X_{q}$. A priori, this lifting need not be $k$-acceptable. However, by using the arguments described in the proof of property $\left(Q_{q}\right)$ of (5.1), one can alter $\tilde{f}$, if necessary, to obtain a $k$-acceptable $q$-lifting of $\rho_{0} z$. This completes the induction.

As a result, we may find a map $F: Y \rightarrow B_{1}(k)$ which induces an isomorphism in $Z_{2}$-cohomology and is therefore a homotopy 2 -equivalence.

COROLlaRY (5.12). Suppose that $Y$ is a spectrum satisfying $H^{*} Y=M_{1}(k)$ such that Theorem (5.1) remains true with $Y$ in place of $B_{1}(k)$. Then $Y$ is homotopy 2-equivalent to $B_{1}(k)$.

Proof. By assumption, there exists $j: Y \rightarrow K\left(Z_{\hat{2}}\right)$ such that $\rho_{0} j: Y \rightarrow K\left(Z_{2}\right)$ represents $1 \in H^{*} Y$. According to (5.8), one can find $\left(Q_{k}, U_{v_{Q_{k}}}\right)$ adapted to $M_{1}(k)$, where $Q_{k}$ is a $(2 k+1)$-dimensional manifold. Since $Y$ satisfies $(5.1), j_{*}: Y_{2 k+1}\left(Q_{k}^{+}\right)$ $\rightarrow H_{2 k+1}\left(Q_{k}^{+} ; Z_{\hat{2}}\right)$ is surjective. Thus, by $S$-duality, $j_{*}: \quad Y^{0}\left(T\left(\nu_{Q_{k}}\right)\right) \rightarrow$ $H^{0}\left(T\left(v_{Q_{k}}\right) ; Z_{\hat{z}}\right)$ is surjective, also. Let $U_{Z}: T\left(v_{Q_{k}}\right) \rightarrow K\left(Z_{\hat{2}}\right)$ represent the Thom class, and choose a $\tilde{U}_{v_{Q_{k}}}: T\left(\nu_{Q_{h}}\right) \rightarrow Y$ such that $j \tilde{U}_{\nu_{Q_{k}}}=U_{Z}$. Then $\rho_{0} j \tilde{U}_{v_{Q_{k}}}=\rho_{0} U_{Z}=$ $U_{v_{Q_{k}}}$. The result now follows directly from (5.11).

Appendix. An inductive definition of $B_{1}(k)$. As mentioned in $\S 2$, the spectra $B_{1}(k)$ were originally defined by Mahowald. In this appendix, we shall propose an alternative, inductive definition based on the techniques of $\S 5$. The spectra that are obtained by this induction will satisfy the properties heretofore ascribed to $B_{1}(k)$, so, in particular, by (5.12), they will be homotopy 2-equivalent to $B_{1}(k)$.

TheOREM (A.1). For each $k \geqslant 0$, there exists a spectrum $\bar{B}(k)$ satisfying the following properties:

(a) $H^{*} \bar{B}(k)=M_{1}(k)$.

(b) There is a map $\bar{i}_{k}: \bar{B}(k) \rightarrow B(k)$ fitting into a cofibration

$$
\bar{B}(k-1) \rightarrow \bar{B}(k) \stackrel{i_{k}}{\rightarrow} B(k)
$$

whose long exact sequence in cohomology realizes (2.13).

(c) Let $\rho_{0}: K\left(Z_{\hat{2}}\right) \rightarrow K\left(Z_{2}\right)$ represent reduction mod 2. Then there exists a map $\bar{j}_{k}$ : $\bar{B}(k) \rightarrow K\left(Z_{\hat{2}}\right)$ such that $\rho_{0} \bar{j}_{k}: \bar{B}(k) \rightarrow K\left(Z_{2}\right)$ represents $1 \in H^{*} \bar{B}(k)$. 
Proof. We shall proceed by induction on $k$.

If $k=0$, define $\bar{B}(0)$ to be the sphere spectrum, completed at 2 . Then $\bar{B}(0)=B(0)$, so let $\overline{i_{0}}=$ identity. Also, define $\overline{j_{0}}: \bar{B}(0) \rightarrow K\left(Z_{\hat{2}}\right)$ to be the 2-completion of a map representing a generator of $H^{0}\left(S^{0} ; Z\right)=Z$.

Next, assume that a spectrum $\bar{B}(k-1)$ has been defined satisfying (A.1). The following analysis relies on the fact that the proofs of $\S 5$ depend only on those properties listed in (A.1). Thus, we may assume that the results of $\S 5$ are valid for $\bar{B}(k-1)$.

Let $\beta: M_{1}(k-1) \rightarrow M(k)$ be the map of $A$-modules defined by $\beta(1)=\mathrm{Sq}^{1}$. The following lemma is the key step in the proof of (A.1).

Lemma (A.2). There is a map $b: B(k) \rightarrow \bar{B}(k-1)$ such that $b^{*}: H^{*} \bar{B}(k-1) \rightarrow$ $H^{*} B(k)$ realizes $\beta$.

Proof OF (A.2). Let $\delta: K\left(Z_{2}\right) \rightarrow K\left(Z_{\hat{2}}\right)$ denote the (degree 1) Bockstein operation associated to the exact sequence of groups

$$
0 \rightarrow Z_{\hat{2}} \stackrel{2}{\rightarrow} Z_{\hat{2}} \stackrel{\rho_{0}}{\rightarrow} Z_{2} \rightarrow 0 .
$$

Now, suppose $\alpha: B(k) \rightarrow K\left(Z_{2}\right)$ represents $1 \in H^{*} B(k)$ and define $z \in$ $H^{1}\left(B(k) ; Z_{\hat{2}}\right)$ by the equation $z=\delta \alpha$. Observe that $\rho_{0} z: B(k) \rightarrow \bar{X}_{0}=K\left(Z_{2}\right)$ represents $\mathrm{Sq}^{1} \in H^{*} B(k)$.

Based on the resolution $\mathscr{C}(k-1)$ of $M_{1}(k-1)$, there is a Postnikov tower $\overline{\mathscr{C}}_{T}$ with limit space $\bar{B}(k-1)$, denoted as follows:

$$
\begin{array}{cccc}
\overline{\mathscr{C}}_{T}: \quad \bar{B}(k-1) & & \\
\vdots & & \\
\downarrow & & \\
\bar{X}_{q} & \rightarrow & \bar{K}_{q+1} \\
\downarrow & & \\
\bar{X}_{q-1} & \rightarrow & \bar{K}_{q} \\
\downarrow & & \\
\vdots & & \\
\downarrow & & \\
\bar{X}_{0} & \rightarrow & \bar{K}_{1}
\end{array}
$$

We will show that, in the language of $\S 5, \rho_{0} z$ lifts all the way up the tower to $\bar{B}(k-1)$ through $(k-1)$-acceptable $q$-liftings. To do this, we again rely on some results of Brown and Peterson.

Let $T_{n}=\left\{a \in A \mid a U_{v_{M}}=0\right.$ for all closed $n$-manifolds $\left.M\right\}$. Brown and Peterson [5] proved that $T_{2 k}=A\left\{\chi\left(\mathrm{Sq}^{i}\right) \mid i>k\right\}$. Thus, by arguments identical to those of (5.8), one can find a $2 k$-dimensional manifold $N$ such that, if $v \in H^{0}\left(T\left(\nu_{N}\right)\right)$ is the Thom class, then

$$
0 \rightarrow A\left\{\chi\left(\mathrm{Sq}^{i}\right) \mid i>k\right\} \rightarrow A \stackrel{v^{*}}{\rightarrow} H^{*}\left(T\left(\nu_{N}\right)\right)
$$


is exact. Next, according to (5.4), there exists a map $\tilde{v}: T\left(\nu_{N}\right) \rightarrow B(k)$ such that $\alpha \tilde{v}=v$. Thus, we have the following commutative diagram:

$$
\begin{aligned}
& \bar{B}(k-1) \\
& \begin{array}{cccc} 
& B(k) & & \vdots \\
\bar{\imath} \nearrow \quad \downarrow \alpha & \searrow \rho_{0} z & \bar{X}_{1}
\end{array} \\
& T\left(v_{N}\right) \quad \underset{r}{\rightarrow} \quad K\left(Z_{2}\right) \quad \underset{\mathrm{Sq}^{1}}{\rightarrow} K\left(Z_{2}\right)=\quad \bar{X}_{0}
\end{aligned}
$$

By induction, there are maps $\bar{i}_{k-1}: \bar{B}(k-1) \rightarrow B(k-1)$ and $\bar{j}_{k-1}: \bar{B}(k-1) \rightarrow$ $K\left(Z_{\hat{2}}\right)$ which induce the obvious maps in cohomology and therefore yield maps of Postnikov towers $\left(\bar{i}_{k-1}\right)_{T}: \overline{\mathscr{C}}_{T} \rightarrow \mathscr{D}_{T}$ and $\left(\bar{j}_{k-1}\right): \overline{\mathscr{C}}_{T} \rightarrow \mathscr{E}_{T}$. Hence, to prove that $\rho_{0} z$ lifts to $\bar{B}(k-1)$, one can simply repeat the proof of (5.11) and thereby obtain the desired map $b: B(k) \rightarrow \bar{B}(k-1)$.

Returning to the proof of (A.1), define $\bar{B}(k)$ to be the fibre of $b$, and let $\bar{i}_{k}$ : $\bar{B}(k) \rightarrow B(k)$ be the inclusion of the fibre. Then properties (a) and (b) follow immediately from the exact sequence (2.13). (Recall that, in the stable category, fibrations and cofibrations are equivalent.)

Note that the map $b$ of (A.2) fits into the following commutative diagram:

$$
\begin{aligned}
& K\left(Z_{\hat{2}}\right) \\
& \downarrow \rho_{0} \\
& \bar{B}(k) \stackrel{i_{k}}{\rightarrow} \quad B(k) \stackrel{\alpha}{\rightarrow} \quad K\left(Z_{2}\right) \\
& \begin{array}{ccc}
b \downarrow & \searrow z & \downarrow \delta \\
\bar{B}(k-1) & \overrightarrow{j_{k-1}} & K\left(Z_{\hat{2}}\right)
\end{array}
\end{aligned}
$$

Since $b \bar{i}_{k}=0, \alpha \bar{i}_{k}$ lifts to a map $\bar{j}_{k}: \bar{B}(k) \rightarrow K\left(Z_{\hat{2}}\right)$ such that $\rho_{0} \bar{j}_{k}=\alpha \bar{i}_{k}$. Then $\bar{j}_{k}$ satisfies property (c).

This completes the induction and thus proves (A.1).

REMARK. Using the lifting techniques of (5.11) and (A.2), one can show that there is a map $h: \bar{B}(2 k) \rightarrow \bar{B}(2 k+1)$ inducing an isomorphism in cohomology; therefore, $\bar{B}(2 k) \simeq \bar{B}(2 k+1)$.

For completeness, we include one final result.

Let $M_{2}$ denote the $Z_{2}$ Moore spectrum. Note that, for purely algebraic reasons, $B(1)=M_{2}$.

LEMMA (A.3). $M_{2} \wedge \bar{B}(2 k) \simeq B(2 k+1)$.

Proof. Recall that $\left\{F_{j}\right\}_{j=0}^{\infty}$ denotes the May filtration of $\Omega^{2} S^{3}$. The structure of this filtration includes products $F_{m} \times F_{n} \rightarrow F_{m+n}$ which, when translated to Thom spectra, yield maps $\mu_{m, n}: B(m) \wedge B(n) \rightarrow B(m+n)$.

Now consider the composition

$$
f: M_{2} \wedge \bar{B}(2 k) \stackrel{\text { id } \wedge \bar{i}_{2 k}}{\rightarrow} B(1) \wedge B(2 k) \stackrel{\mu_{1,2 k}}{\rightarrow} B(2 k+1) .
$$


It is easy to check that $f$ induces an isomorphism in cohomology and hence must be a homotopy equivalence.

Upon careful review, one finds that our prior findings about $B_{1}(k)$ really depended only on naturality arguments involving the maps $i_{k}: B_{1}(k) \rightarrow B(k)$ and $j$ : $B_{1}(k) \rightarrow K\left(Z_{\hat{2}}\right)$; also, the computation of $\pi_{*} B_{1}(k)$ made use of the fact that $M_{2} \wedge B_{1}(2 k) \simeq B(2 k+1)$. But, according to (A.1) and (A.3), the spectra $\bar{B}(k)$ possess completely analogous properties. Hence, our results on $B_{1}(k)$ all go over to $\bar{B}(k)$ with exactly the same proofs. In other words, we could have initially treated $\bar{B}(k)$ as our basic object of study without any loss.

\section{REFERENCES}

1. A. K. Bousfield et al., The mod-p lower central series and the Adams spectra sequence, Topology 5 (1966), 331-342.

2. A. K. Bousfield and D. M. Kan, Homotopy limits, completions and localizations, Lecture Notes in Math., vol. 304, Springer-Verlag, New York, 1972.

3. E. H. Brown, Jr., and S. Gitler, A spectrum whose cohomology is a certain ryclic module over the Steenrod algebra, Topology 12 (1973), 283-295.

4. E. H. Brown, Jr., and F. P. Peterson, The Brown-Gitler spectrum, $\Omega^{2} S^{3}$, and $\eta_{j} \in \pi_{2^{\prime}}(S)$ (preprint).

5. __ Relations among characteristic classes. I, Topology 3 (1964), 39-52.

6. On the stable decomposition of $\Omega^{2} S^{r+2}$. Trans. Amer. Math. Soc. 243 (1978), 287-298.

7. F. R. Cohen, T. J. Lada and J. P. May, The homologl of iterated loop spaces, Lecture Notes in Math., vol. 533, Springer-Verlag, New York, 1976.

8. F. R. Cohen, J. P. May and L. R. Taylor, $K(Z, 0)$ and $K\left(Z_{2}, 0\right)$ as Thom spectra, Illinois J. Math. 25 (1981), 99-106.

9. R. L. Cohen, The geometry of $\Omega^{2} S^{3}$ and braid orientations, Invent. Math. 54 (1979), 53-67.

10. D. M. Davis, S. Gitler and M. Mahowald, The stable geometric dimension of vector bundles oter real projective spaces, Trans. Amer. Math. Soc. 268 (1981), 39-61.

11. P. Goerss, Results on Brown-Gitler type spectra, Ph. D. Dissertation, M. I. T., 1983.

12. I. M. James, Reduced product spaces, Ann. of Math. (2) 62 (1955), 170-197.

13. M. Mahowald, bo-resolutions, Pacific J. Math. 92 (1981), 365-383.

14. _ A new infinite family in ${ }_{2} \pi_{*}^{S}$. Topology 16 (1977), 249-256.

15. __ Ring spectra which are Thom complexes, Duke Math. J. 46 (1979), 549-559.

16. J. P. May, The geometry of iterated loop spaces, Lecture Notes in Math., vol. 271, Springer-Verlag, New York, 1972.

17. J. Milnor, The Steenrod algebra and its dual, Ann. of Math. (2) 67 (1958), 150-171.

18. N. E. Steenrod and D. B. A. Epstein, Cohomology operations. Ann. of Math. Studies, no. 50. Princeton Univ. Press, Princeton, N. J., 1962.

Department of Mathematics, Bowdoin College, Brunswick, Maine 04011

Current address: Department of Mathematics, Haverford College, Haverford, Pennsylvania 19041 